2. To: (Receiving Organization)

DISTRIBUTION

5. Proj./Prog./Dept./Div.:

HANDI 2000

8. Originator Remarks: COSTING, BUSINESS SYSTEM IMPLEMENTATION, FINANCE, AS-IS/TO-BE

11. Receiver Remarks:
KEY WORDS: H2K, BMS, HANDI 2000, PEOPLESOFT. GENERAL LEDGER, PROJECT
3. From: (Originating Organization) SYSTEMS FLUOR DANIEL HANFORD 6. Design Authority/Design Agent/Cog. Engr.:

DAWN E. ADAMS
4. Related EDT No::

N/A

7. Purchase Order No:

$\mathrm{N} / \mathrm{A}$

9. Equip./Component No.

$\mathrm{N} / \mathrm{A}$

10. System/Bldg./Facility:

$\mathrm{N} / \mathrm{A}$

12. Major Assm. Dwg. No.:

$\mathrm{N} / \mathrm{A}$

13. Permit/Permit Application No.

$\mathrm{N} / \mathrm{A}$

14. Required Response Date:

$\mathrm{N} / \mathrm{A}$

\begin{tabular}{|c|c|c|c|}
\hline (F) & $(\mathrm{G})$ & $(\mathrm{H})$ & $(\mathrm{I})$ \\
\hline $\begin{array}{c}\text { Approval } \\
\text { Desig- } \\
\text { nator }\end{array}$ & $\begin{array}{c}\text { Reason } \\
\text { frans- } \\
\text { mittal }\end{array}$ & $\begin{array}{c}\text { Origi- } \\
\text { nator } \\
\text { Dispo- } \\
\text { stion }\end{array}$ & $\begin{array}{c}\text { Receiv- } \\
\text { er } \\
\text { Dispo- } \\
\text { sition }\end{array}$ \\
\hline N/A & 2 & & \\
\hline N/A & 2 & & \\
\hline N/A & 2 & & \\
\hline & & & \\
\hline
\end{tabular}

(SEE SHEET 2)

16.

DATA TRANSMITTED

\begin{tabular}{|l|l|}
\hline $\begin{array}{l}\text { (A) } \\
\text { Ifem } \\
\text { No. }\end{array}$ & (B) DocumentDrawing No. \\
\hline 1 & HNF - 2640 \\
\hline & HNF-2641 \\
\hline 2 & HNF-2642 \\
\hline 3 & \\
\hline & (SEE SHEET 2) \\
\hline
\end{tabular}

KEY

\begin{tabular}{|c|c|c|c|c|}
\hline Approval Designator (F) & \multicolumn{2}{|c|}{ Reason for Transmittal (G) } & \multicolumn{2}{|c|}{ Disposition $(\mathrm{H})$ \& (l) } \\
\hline $\begin{array}{l}\text { E. S, Q, D OR N/A } \\
\text { (See WHC-CM-3-5, } \\
\text { Sec. 12.7) }\end{array}$ & $\begin{array}{l}\text { 1. Approval } \\
\text { 2. Release } \\
\text { 3. Information }\end{array}$ & $\begin{array}{l}\text { 4. Review } \\
\text { 5. Post-Review } \\
\text { 6. Dist. (Receipt Acknow. Required) }\end{array}$ & $\begin{array}{l}\text { 1. Approved } \\
\text { 2. Approved w/comment } \\
\text { 3. Disapproved w/comment }\end{array}$ & $\begin{array}{l}\text { 4. Reviewed no/comment } \\
\text { 5. Reviewed w/comment } \\
\text { 6. Recelpt acknowledged }\end{array}$ \\
\hline
\end{tabular}

17.

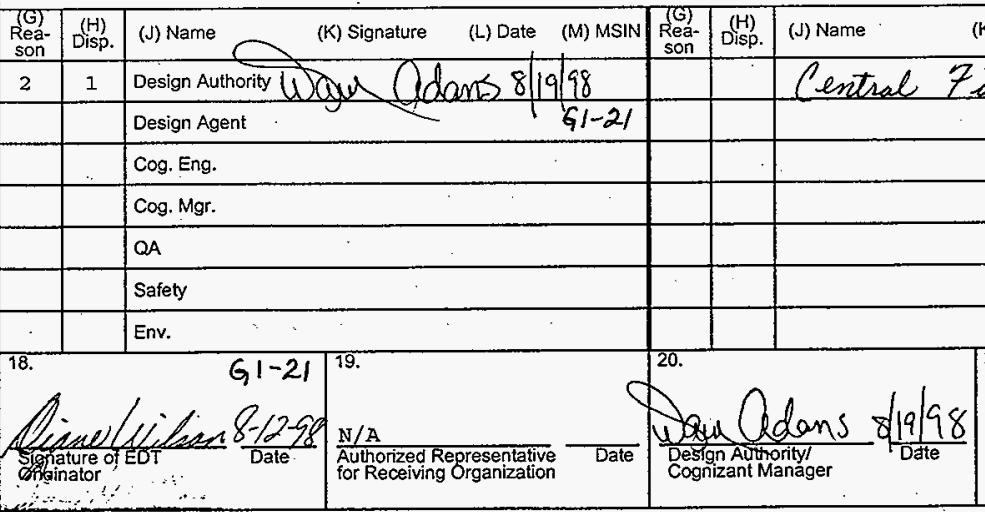

21. DOE APPROVAL (if required)

Ctrl No.

$\square$ Approved

$\square$ Approved w/comments

$\square$ Disapproved w/comments 


\section{ENGINEERING DATA TRANSMITTAL}

(CONTINUATION PAGE)

5. Proj.JProg./Dept.Div.:

HANDI 2000
6. Cog. Eng.

DAWN E. ADAMS
1. EDT

625358
4
15.

(A)
Item

No.

\section{DATA TRANSMITTED}

\begin{tabular}{|c|c|}
\hline $\begin{array}{c}\text { (C) } \\
\text { Sheet } \\
\text { No. }\end{array}$ & $\begin{array}{c}\text { (D) } \\
\text { Rev. } \\
\text { No. }\end{array}$ \\
\hline N/A & 0 \\
\hline & \\
\hline
\end{tabular}

\begin{tabular}{|l|l|}
\hline & \\
\hline &. \\
\hline
\end{tabular}

$5 \quad$ HNF-2643

$6 \quad$ HNF -2644

7 HNF-2645

8 HINF-2646

$9 \quad$ HNF -2647

$10 \quad$ HNF -2648

11 HINF - 2649

12 HNF -2650

13 HNF - 2651
(E).Title or Description of Data Transmitted

FIT GAP ANALYSIS FOR

HANDI 2000 BUSINESS

MANAGEMENT SYSTEM

CLOSING PROCEDURE FOR

HANDI 2000 BUSINESS

MANAGEMENT SYSTEM

FIXED ASSETS ACCOUNTING

FOR HANDI 2000 BUSINESS

MANAGEMENT SYSTEM

\begin{tabular}{ll|l|l} 
FUNDS CONTROL FOR HANDI & N/A & 2
\end{tabular}

2000 BUSINESS MANAGEMENT

SYSTEM

GL CHART OF ACCOUNTS

MAINTENANCE FOR HANDI

2000 BUSINESS MANAGEMENT

SYSTEM

HANFORD BUSINESS

STRUCTURE FOR BUSINESS

MANAGEMENT SYSTEM

INVENTORY PROCESSES

FOR HANDI 2000 BUSTNESS

MANAGEMENT SYSTEM

INVOICE/DISCAS FOR HANDI

2000 BUSINESS MANAGEMENT

SYSTEM

LABOR COSTING FOR HANDI

2000 BUSINESS MANAGEMENT

SYSTEM

PASSPORT-PEOPLESOFT

INTEGRATION FOR HANDI

2000 BUSINESS MANAGEMENT

SYSTEM

\begin{tabular}{|c|c|c|c|}
\hline (F) & (G) & (H) & (I) \\
\hline $\begin{array}{c}\text { Approval } \\
\text { Desig- } \\
\text { nator }\end{array}$ & $\begin{array}{c}\text { Reason } \\
\text { fror } \\
\text { Trans- } \\
\text { mittal }\end{array}$ & $\begin{array}{c}\text { Origi- } \\
\text { nator } \\
\text { Dispo- } \\
\text { stion }\end{array}$ & $\begin{array}{c}\text { Receiv- } \\
\text { er } \\
\text { Dispo- } \\
\text { sition }\end{array}$ \\
\hline N/A & 2 & & \\
\hline & & & \\
\hline N/A & 2 & & \\
\hline & & & \\
\hline N/A & 2 & & \\
\hline & & & \\
\hline N/A & 2 & & \\
\hline & & & \\
\hline & & & \\
\hline N/A & 2 & & \\
\hline & & & \\
\hline & & & \\
\hline N/A & 2 & & \\
\hline & 2 & & \\
\hline & & & \\
\hline & & & \\
\hline & & & \\
\hline & & & \\
\hline & & & \\
\hline & & & \\
\hline & & & \\
\hline & & & \\
\hline & & & \\
\hline & & & \\
\hline & & & \\
\hline & & & \\
\hline & & & \\
\hline & & & \\
\hline
\end{tabular}

\begin{tabular}{|l|l|l|l|}
\hline $\begin{array}{c}\text { Approval } \\
\text { Desig- } \\
\text { nator }\end{array}$ & $\begin{array}{c}\text { Reason } \\
\text { for } \\
\text { Trans- } \\
\text { mittal }\end{array}$ & $\begin{array}{c}\text { Origi- } \\
\text { nator } \\
\text { Dispo- } \\
\text { stion }\end{array}$ & $\begin{array}{c}\text { Recei } \\
\text { Er } \\
\text { Dispo- } \\
\text { sition }\end{array}$ \\
\hline N/A & 2 & & \\
\hline N/A & 2 & & \\
\hline & & & \\
\hline N/A & 2 & & \\
\hline & & & \\
\hline & & & \\
\hline N/A & 2 & & \\
\hline & & & \\
\hline N/A & 2 & & \\
\hline & & & \\
\hline N/A & 2 & & \\
\hline & & & \\
\hline & & & \\
\hline & & & \\
\hline & & & \\
\hline & & & \\
\hline & & & \\
\hline & & & \\
\hline & & & \\
\hline & & & \\
\hline & & & \\
\hline
\end{tabular}

\begin{tabular}{l|l}
$\mathrm{N} / \mathrm{A}$ & 2 \\
\hline
\end{tabular}

\begin{tabular}{l|l} 
N $/ \mathrm{A}$ & 2 \\
\hline
\end{tabular}

\begin{tabular}{|l|l|l|l|}
\hline $\begin{array}{c}\text { Approval } \\
\text { Desig- } \\
\text { nator }\end{array}$ & $\begin{array}{c}\text { Reason } \\
\text { for } \\
\text { Trans- } \\
\text { mittal }\end{array}$ & $\begin{array}{c}\text { Origi- } \\
\text { nator } \\
\text { Dispo- } \\
\text { stion }\end{array}$ & $\begin{array}{c}\text { Recei } \\
\text { Er } \\
\text { Dispo- } \\
\text { sition }\end{array}$ \\
\hline N/A & 2 & & \\
\hline N/A & 2 & & \\
\hline & & & \\
\hline N/A & 2 & & \\
\hline & & & \\
\hline & & & \\
\hline N/A & 2 & & \\
\hline & & & \\
\hline N/A & 2 & & \\
\hline & & & \\
\hline N/A & 2 & & \\
\hline & & & \\
\hline & & & \\
\hline & & & \\
\hline & & & \\
\hline & & & \\
\hline & & & \\
\hline & & & \\
\hline & & & \\
\hline & & & \\
\hline & & & \\
\hline
\end{tabular}

\begin{tabular}{|l|l|l|l|}
\hline $\begin{array}{c}\text { Approval } \\
\text { Desig- } \\
\text { nator }\end{array}$ & $\begin{array}{c}\text { Reason } \\
\text { for } \\
\text { Trans- } \\
\text { mittal }\end{array}$ & $\begin{array}{c}\text { Origi- } \\
\text { nator } \\
\text { Dispo- } \\
\text { stion }\end{array}$ & $\begin{array}{c}\text { Recei } \\
\text { Er } \\
\text { Dispo- } \\
\text { sition }\end{array}$ \\
\hline N/A & 2 & & \\
\hline N/A & 2 & & \\
\hline & & & \\
\hline N/A & 2 & & \\
\hline & & & \\
\hline & & & \\
\hline N/A & 2 & & \\
\hline & & & \\
\hline N/A & 2 & & \\
\hline & & & \\
\hline N/A & 2 & & \\
\hline & & & \\
\hline & & & \\
\hline & & & \\
\hline & & & \\
\hline & & & \\
\hline & & & \\
\hline & & & \\
\hline & & & \\
\hline & & & \\
\hline & & & \\
\hline
\end{tabular}

\begin{tabular}{|l|l|l|l|}
\hline $\begin{array}{c}\text { Approval } \\
\text { Desig- } \\
\text { nator }\end{array}$ & $\begin{array}{c}\text { Reason } \\
\text { for } \\
\text { Trans- } \\
\text { mittal }\end{array}$ & $\begin{array}{c}\text { Origi- } \\
\text { nator } \\
\text { Dispo- } \\
\text { stion }\end{array}$ & $\begin{array}{c}\text { Recei } \\
\text { Er } \\
\text { Dispo- } \\
\text { sition }\end{array}$ \\
\hline N/A & 2 & & \\
\hline N/A & 2 & & \\
\hline & & & \\
\hline N/A & 2 & & \\
\hline & & & \\
\hline & & & \\
\hline N/A & 2 & & \\
\hline & & & \\
\hline N/A & 2 & & \\
\hline & & & \\
\hline N/A & 2 & & \\
\hline & & & \\
\hline & & & \\
\hline & & & \\
\hline & & & \\
\hline & & & \\
\hline & & & \\
\hline & & & \\
\hline & & & \\
\hline & & & \\
\hline & & & \\
\hline
\end{tabular}

\begin{tabular}{|l|l|l|l|}
\hline $\begin{array}{c}\text { Approval } \\
\text { Desig- } \\
\text { nator }\end{array}$ & $\begin{array}{c}\text { Reason } \\
\text { for } \\
\text { Trans- } \\
\text { mittal }\end{array}$ & $\begin{array}{c}\text { Origi- } \\
\text { nator } \\
\text { Dispo- } \\
\text { stion }\end{array}$ & $\begin{array}{c}\text { Recei } \\
\text { Er } \\
\text { Dispo- } \\
\text { sition }\end{array}$ \\
\hline N/A & 2 & & \\
\hline N/A & 2 & & \\
\hline & & & \\
\hline N/A & 2 & & \\
\hline & & & \\
\hline & & & \\
\hline N/A & 2 & & \\
\hline & & & \\
\hline N/A & 2 & & \\
\hline & & & \\
\hline N/A & 2 & & \\
\hline & & & \\
\hline & & & \\
\hline & & & \\
\hline & & & \\
\hline & & & \\
\hline & & & \\
\hline & & & \\
\hline & & & \\
\hline & & & \\
\hline & & & \\
\hline
\end{tabular}

\begin{tabular}{l|l}
$\mathrm{N} / \mathrm{A}$ & 2 \\
\hline
\end{tabular}

\begin{tabular}{|l|l|l|l|}
\hline & & & \\
\hline$N / A$ & 2 & & \\
\hline & & & \\
\hline & & & \\
\hline N/A & 2 & & \\
\hline & & & \\
\hline & & & \\
\hline & & & \\
\hline & & & \\
\hline
\end{tabular}


(CONTINUATION PAGE)

5. Proj./Prog./Dept.Div.:

HANDI 2000
6. Cog. Eng.

DAWN E. ADAMS
1. EDT

625358

Page 3 of 4

15.

(A)
item

Nom

(B) DocumentDrawing No.

14

HNE -2652

\begin{tabular}{|l|l}
\hline & \\
\hline & \\
\hline 15 & HNF-2653 \\
\hline
\end{tabular}

\begin{tabular}{|l|l}
\hline 15 & HNF - 2653 \\
\hline &
\end{tabular}

16 FNN -2654

\begin{tabular}{|l|l|}
\hline & \\
\hline & \\
\hline & \\
\hline & \\
\hline
\end{tabular}

17 HNNF -2655

\begin{tabular}{|l|l|}
\hline & \\
\hline & \\
\hline & \\
\hline
\end{tabular}

18 HNN -2656$$
\text { 西 }
$$ 


\begin{tabular}{|c|c|c|c|c|c|c|c|c|}
\hline \multicolumn{9}{|c|}{$\begin{array}{c}\text { ENGINEERING DATA TRANSMITTAL } \\
\text { (CONTINUATION PAGE) }\end{array}$} \\
\hline \multicolumn{2}{|c|}{$\begin{array}{l}\text { 5. Proj./Prog.Joept.Div.: } \\
\text { HANDI } 2000\end{array}$} & \multicolumn{3}{|c|}{$\begin{array}{l}\text { 6. Cog. Eng. } \\
\text { DAWN E. ADAMS }\end{array}$} & \multicolumn{2}{|l|}{$\begin{array}{r}\text { 1. EDT } \\
625358\end{array}$} & \multicolumn{2}{|c|}{ Page 4 of 4} \\
\hline \multicolumn{2}{|l|}{15.} & \multicolumn{3}{|c|}{ DATA TRANSMITTED } & (F) & (G) & (H) & (l) \\
\hline $\begin{array}{l}\text { (A) } \\
\text { Item } \\
\text { No. }\end{array}$ & (B) Document/Drawing No. & $\begin{array}{l}\text { (C) } \\
\text { Sheet } \\
\text { No. }\end{array}$ & $\begin{array}{l}\text { (D) } \\
\text { Rev. } \\
\text { No. }\end{array}$ & $\begin{array}{l}\text { (E) Title or Description of Data } \\
\text { Transmitted }\end{array}$ & $\begin{array}{l}\text { Approval } \\
\text { Desig- } \\
\text { nator }\end{array}$ & $\begin{array}{l}\text { Reason } \\
\text { for } \\
\text { Trans- } \\
\text { mittal }\end{array}$ & $\begin{array}{c}\text { Origi- } \\
\text { nator } \\
\text { Dispo- } \\
\text { stion } \\
\end{array}$ & \begin{tabular}{|c|} 
Receiv- \\
er \\
Dispo- \\
sition \\
\end{tabular} \\
\hline \multirow[t]{4}{*}{23} & HNF -2663 & $\mathrm{~N} / \mathrm{A}$ & 0 & WALK-IN WORK FOR OTHER & $\mathrm{N} / \mathrm{A}$ & 2 & & \\
\hline & & & & HANFORD CONTRACTORS FOR & & & & \\
\hline & & & & HANDI 2000 BUSINESS & & & & \\
\hline & & & & MANAGEMENT SYSTEM & & & & \\
\hline \multirow[t]{4}{*}{24} & HNF -2664 & $\mathrm{~N} / \mathrm{A}$ & 0 & DOE HANFORD REQUEST FOR & & & & \\
\hline & & & & SERVICES FOR HANDI 2000 & & & & \\
\hline & & & & BUS INESS MANAGEMENT & & & & \\
\hline & & & & SYSTEM & & & & \\
\hline & & & & & & & & \\
\hline & & & & & & & $\cdot$ & \\
\hline & & & & & & & & \\
\hline & & & . & & & & & \\
\hline & & & & & & & & \\
\hline & & & & & & & & \\
\hline & & & & & & & & \\
\hline & & & & & & & & \\
\hline & & & & & & & & \\
\hline & . & & & & & & & \\
\hline & & & & & & & & \\
\hline & & & & & & & & \\
\hline & & & & & & & & \\
\hline & & & & & & & & \\
\hline & & & & & & & & \\
\hline & & & & & & & & \\
\hline & & & & & & & & \\
\hline & & & & & & & & \\
\hline & & & & & & & & \\
\hline & . & & & & & & & \\
\hline & & & & & & & & \\
\hline & & & & & & & & \\
\hline & & & & & & & & \\
\hline & & & & & & & & \\
\hline & & & & & & & & \\
\hline
\end{tabular}




\title{
FIT GAP ANALYSIS FOR HANDI 2000 BUSINESS MANAGEMENT SYSTEM
}

\author{
Diane Wilson, Fluor Daniel Hanford Co. \\ MSN $31-22$ \\ Richland, WA 99352 \\ U.S. Department of Energy Contract DE-AC06-96RL13200

$\begin{array}{llll}\text { EDT/ECN: } 625358 & \text { UC: } 900 & \\ \text { Org Code: } & \text { FK260000 } & \text { Charge Code: } & \text { YBPME } \\ \text { B\&R Code: } & \text { EW7070100 } & \text { Total Pages: } \frac{326}{\sqrt{6}} & 35\end{array}$

Key Words: CHARTS, STANDARDS LAB (FROM SLC), GRAPHICS (FROM HUB), OCCUPANCY (from manual entry), Contracts Accrual, Material, Labor, MPR, Allocations Methodology, BMS Process Flow, Transactional Journal Entries.

Abstract: The purpose of the Fit Gap Analysis sessions was to identify any significant functional gaps with Peoplesoft Financials as it relates to the business requirements of Fluor Daniel Hanford (FDH). To that end, a series of prototyping sessions were held with members of the core team and subject matter experts, where functionality and test cases were discussed. Test cases were developed for Peoplesoft projects and General Ledger and run through the data processing model.

TRADEMARK DISCLAIMER. Reference herein to any specific commercial product, process, or service by trade name, trademark, manufacturer, or otherwise, does not necessarily constitute or imply its endorsement, recommendation, or favoring by the United States Government or any agency thereof or its contractors or subcontractors.

Printed in the United States of America. To obtain copies of this document, contact: Document Control Services, P.O. Box 950, Mailstop H6-08, Richland WA 99352, Phone (509) 372-2420; Fax (509) 376-4989.

PeopleSoft is a trademark of Indus Corp. Passport is a trademark of Indus corp.
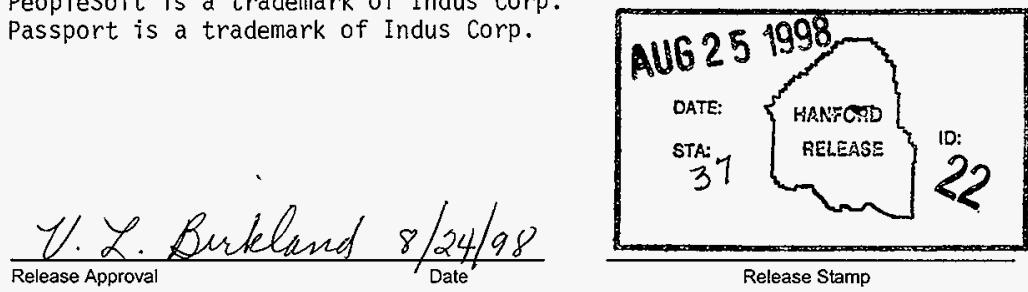

Release Stamp 


\section{Memorandum}

To:
D.E. Adams
G1-21
M.J. Byrd
$\mathrm{H} 2-08$,
A.A. Carter
$\mathrm{H} 2-19$,
C.R. Hopkins G1-57,

K.B. Adamsons H2-19

R.C. Corless H2-19,

J.A. Caldwell G1-22,

R.J. McFadzean H2-19

From: P. C. Felts

Date: April 17, 1998

Re: PeopleSoft General Ledger / Project Costing -- Business System Implementation Sessions Final Documentation

\section{HANDI 2000 - Finance}

Attached for your information is the complete set of documentation of the work flow analysis from the Business System Implementation (BSI) Sessions.

One of the goals of finalizing documentation from the BSI Sessions is to use the documents as a communication tool.

Other goals of the BSI sessions were to validate the As-Is process, and describe the vision of the To-Be process that fit the baseline software processes. In the future, this documentation will be accessible electronically.via a read-only shared area.

I thank you and your staff for your support during this information gathering and envisioning stage of the new system implementation project

If you have any questions, I can be reached on 376-0367 or via email. 
Date:

$4 / 17 / 98$

Documentation: Finance Business System Implementation (BSI) Sessions

\section{Processes Included:}

- Accrual

- Allocations

- Budget

- Fit-Gap Analysis

- Closing Procedure

- Fixed Assets Accounting

- Funds Control

- General Ledger Account Maintenance

- Hanford Business Structure

- Inventory Processes

- Invoice/DISCAS

- Labor Costing

- Passport-PeopleSoft Integration

- Unit Billed Service

- Travel \& Treasury:

- OTS Cash Disbursement Process

- Travel Expense Reimbursement

- Cash Receipt Process .

_ Savings, Pensions, \& Insurance:

_ Insurance Payment Process

- Pension Payment Process

- Savings Plan Payment Process

- Other Hanford Contractors:

- Worked Performed by Enco's for PHMC

- Worked Performed by Enco's for OHC

- Worked Performed by PHMC for OHC - Work Order

- Worked Performed by OHC for PHMC - Work Order Process

- Worked Performed by PHMC for OHC - Walk In

- Worked Performed by PHMC for DOE-Richland using the Request for Service Process

FDH Project Lead:

Name

Signature

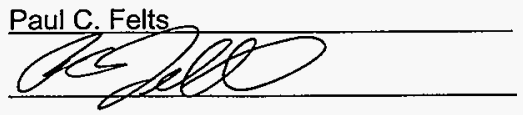




\section{Fit Gap Analysis}

\section{Overview}

The purpose of the Fit Gap Analysis sessions was to identify any significant functional gaps with PeopleSoft Financials as it relates to the business requirements of Fluor Daniel Hanford (FDH). To that end, a series of prototyping sessions were held with members of the core team and subject matter experts, where functionality and test cases were discussed. Test cases were developed for PeopleSoft Projects and General Ledger and run through the data processing model. The results of the sessions were discussed and evaluated with members of the prototyping team.

\section{Assumptions}

1. All systems (excepting Payroll and Passport) feed PeopleSoft Projects which then feeds PeopleSoft General Ledger. There will be a separate feed from Payroll to update the General Ledger. Passport will generate a dual entry to Projects and General Ledger.

2. The interfacing systems would feed data in the same format that it was loaded manually into Projects.

3. The business cases developed by FDH were a complete set of transaction types.

4. Labor will use 92+ Categories and 4 Sub Categories for transaction classification (BUS, PTB, ABS, COS).

5. Accruals will have their own Analysis Type, as will Reversals (ACR, RVR).

\section{Test Case Scenarios}

Several business cases were developed by the core team. The expense information from the case was manually entered into PeopleSoft Projects in the same format it is expected to be received from the generating feeder system. The expense information was then forwarded as a double sided entry from PeopleSoft Projects to PeopleSoft General Ledger. We tested the following seven different types of transactions:

1. Standards Lab-emulating a feed from SLC. This transaction transfers the costs incurred by the Standards Lab to the direct projects that utilized Standard's Lab's services that month.

2. Graphics-emulating a feed from HUB. This transaction transfers the costs incurred by the Graphics department to the direct projects that utilized Graphic's services that month.

3. Occupancy-emulating a manual entry to Projects. This transaction allocates the costs of maintaining space to the projects occupying space.

4. Contracts accruals-emulating a feed from Passport.

5. Materia//Contracts- emulating a feed from Passport.

6. Labor- emulating a feed from TIS/Payroll. This transaction assigns the costs of labor to the project that the employee worked on for the pay period ending the week prior

7. MPR allocation- emulating a transfer of indirect costs between projects. This transaction tranfers the costs associated with purchasing (i.e. labor, materials, overhead) to the direct projects that purchased materials, liquidating cost accumulated in the indirect purchasing project. This was done both in the General Ledger and in Projects.

\section{Issues}

1. Need chart of accounts to be finalized and approved.

2. Does the panel reference to "activity" need to be changed to "code of account"?

3. How to reverse an allocation alone only in Projects.

4. For offset entries to Projects, do we use a Code of Account, a Resource Type or an Analysis Type?

\section{Next Steps}


No significant functional gaps were identified based on the business cases tested.

\section{Appendicies}

\section{Standards Lab (from SLC)}

\begin{tabular}{|c|c|c|c|c|c|}
\hline Project ID & $\mathrm{COA}$ & Dept ID & Res Type & Res Categ & Total \$ \\
\hline 100001 & $\overline{\mathrm{A} 001}$ & 10539 & Dirct & SLC & 500 \\
\hline 100003 & A005 & 32592 & Dirct & SLC & 2000 \\
\hline 100004 & $\mathrm{D} 002$ & 39754 & Dirct & SLC & 1500 \\
\hline 100004 & D002 & 92734 & Dirct & SLC & 3000 \\
\hline 200001 & E003 & 47610 & Dirct & SLC & 4500 \\
\hline 200002 & F004 & 47610 & Dirct & SLC & 2000 \\
\hline 200003 & G008 & 47610 & Dirct & SLC & 400 \\
\hline 300001 & K001 & 42801 & Dirct & SLC & 800 \\
\hline 300003 & No01 & 42801 & Dirct & SLC & 100 \\
\hline 300004 & P001 & 42801 & Dirct & SLC & 1300 \\
\hline 400002 & Do02 & 10539 & Dirct & SLC & 1400 \\
\hline 400003 & E003 & 34912 & Dirct & SLC & 2400 \\
\hline 400004 & F004 & 34912 & Dirct & SLC & 3400 \\
\hline 400005 & G008 & 34912 & Dirct & SLC & 1500 \\
\hline 400006 & K001 & 39754 & Dirct & SLC & 1500 \\
\hline 500001 & L002 & 92734 & Dirct & SLC & 9000 \\
\hline 500003 & P001 & 92734 & Dirct & SLC & 1700 \\
\hline 500004 & $\mathrm{~A} 001$ & 10539 & Dirct & SLC & 900 \\
\hline 500005 & $\mathrm{~A} 004$ & 92734 & Dirct & SLC & 600 \\
\hline 500006 & $A 005$ & 47610 & Dirct & SLC & 500 \\
\hline 500007 & D002 & 39754 & Dirct & SLC & 1100 \\
\hline 500008 & $\mathrm{E} 003$ & 39754 & Dirct & SLC & 2000 \\
\hline 500010 & G008 & 32592 & Dirct & SLC & 2300 \\
\hline 500011 & K001 & 34912 & Dirct & SLC & 200 \\
\hline 500015 & $A 001$ & 42801 & Dirct & SLC & 1500 \\
\hline 800001 & $\mathrm{~A} 004$ & 32592 & Dirct & SLC & 300 \\
\hline 800002 & $A 005$ & 92734 & Dirct & SLC & 1100 \\
\hline 900002 & F004 & 10539 & Dirct & SLC & 300 \\
\hline
\end{tabular}

\section{Graphics (from HUB)}

\begin{tabular}{|c|c|c|c|c|c|}
\hline Project ID & $\mathrm{COA}$ & Dept ID & Res Type & Res Categ & Total \$ \\
\hline 100001 & $\overline{\mathrm{A} 001}$ & 10539 & Dirct & Graph & 1000 \\
\hline 200003 & G008 & 47610 & Dirct & Graph & 1500 \\
\hline 300001 & K001 & 42801 & Dirct & Graph & 2000 \\
\hline 400005 & G008 & 34912 & Dirct & Graph & 2500 \\
\hline 400006 & K001 & 39754 & Dirct & Graph & 3000 \\
\hline 500001 & L002 & 92734 & Dirct & Graph & $350 c$ \\
\hline 500002 & N001 & 10539 & Dirct & Graph & 4000 \\
\hline 800001 & A004 & 32592 & Dirct & Graph & 4500 \\
\hline 900001 & E003 & 10539 & Dirct & Graph & 5000 \\
\hline
\end{tabular}

\section{Occupancy (from manual entry)}

$\frac{\text { Project ID }}{800001} \quad \frac{\text { COA }}{\text { A004 }} \quad \frac{\text { Dept ID }}{32592} \quad \frac{\text { Res Type Res Categ }}{\text { Dirct }} \frac{\text { Total } \$}{\text { Occup }}$


HANDI Fluor Daniel Hanford - PeopleSoft Financials

$\begin{array}{llllll}800002 & \text { A004 } & 92734 & \text { Dirct } & \text { Occup } & 2500 \\ 800003 & \text { A004 } & 10539 & \text { Dirct } & \text { Occup } & 3000 \\ 900001 & \text { A004 } & 10539 & \text { Dirct } & \text { Occup } & 3000 \\ 900002 & \text { A004 } & 10539 & \text { Dirct } & \text { Occup } & 3500 \\ 900003 & \text { A004 } & 92734 & \text { Dirct } & \text { Occup } & 3500 \\ 900004 & \text { A004 } & 92734 & \text { Dirct } & \text { Occup } & 4000 \\ 900004 & \text { A004 } & 10539 & \text { Dirct } & \text { Occup } & 4000 \\ 900006 & \text { A004 } & 10539 & \text { Dirct } & \text { Occup } & 5000\end{array}$

4. Contracts Accrual (from Passport)

\begin{tabular}{|c|c|c|c|c|c|c|c|}
\hline Project ID & $\mathrm{COA}$ & Dept ID & Res Type & $\frac{\text { Res }}{\text { Categ }}$ & $\mathrm{PO} \#$ & Line & Total $\$$ \\
\hline 400002 & D002 & 10539 & Subcn & & M12345 & 001 & 4000 \\
\hline 500002 & No01 & 10539 & Subcn & & M99000 & 001 & 4000 \\
\hline 500004 & $A 001$ & 10539 & Subcn & & M77000 & 001 & 4000 \\
\hline 500013 & N001 & 10539 & Subcn & & M12321 & 001 & 4000 \\
\hline 800003 & D002 & 10539 & Subcn & & M15853 & 001 & 4000 \\
\hline 900001 & E003 & 10539 & Suben & & M12673 & 001 & 4000 \\
\hline 900002 & F004 & 10539 & Subcn & & M98467 & 001 & 4000 \\
\hline 900004 & K001 & 10539 & Subcn & & M07777 & 001 & 4000 \\
\hline 900006 & N001 & 10539 & Subcn & & M04444 & 001 & 4000 \\
\hline 900007 & P001 & 10539 & Suben & & M00333 & 001 & 4000 \\
\hline 500009 & F004 & 32592 & Subcn & & M22000 & 001 & 4000 \\
\hline 500010 & G008 & 32592 & Suben & & M34543 & 001 & 4000 \\
\hline 800001 & A004 & 32592 & Subcn & & M00990 & 001 & 4000 \\
\hline 400003 & E003 & 34912 & Suben & & M23456 & 001 & 4000 \\
\hline 400004 & F004 & 34912 & Subcn & & M34567 & 001 & 4000 \\
\hline 400005 & G008 & 34912 & Suben & & M45678 & .001 & 4000 \\
\hline 500011 & K001 & 34912 & Subcn & & M56765 & 001 & 4000 \\
\hline 500012 & $\mathrm{~L} 002$ & 34912 & Suben & & M89098 & 001 & 4000 \\
\hline 400006 & K001 & 39754 & Subcn & & M56789 & 001 & 4000 \\
\hline 500007 & D002 & 39754 & Subcn & & M44000 & 001 & 4000 \\
\hline 500008 & E003 & 39754 & Subcn & & M33000 & 001 & 4000 \\
\hline 500014 & P001 & 42801 & Suben & & M67766 & 001 & 4000 \\
\hline 500015 & $\mathrm{~A} 001$ & 42801 & Subcn & & M34433 & 001 & 4000 \\
\hline 500006 & A005 & 47610 & Subcn & & M55000 & 001 & 4000 \\
\hline 500001 & L002 & 92734 & Subcn & & M67890 & 001 & 4000 \\
\hline 500003 & P001 & 92734 & Subch & & M88000 & 001 & 4000 \\
\hline 500005 & $\mathrm{~A} 004$ & 92734 & Subcn & & M66000 & 001 & 4000 \\
\hline 800002 & A005 & 92734 & Suben & & M53708 & 001 & 4000 \\
\hline 900003 & G008 & 92734 & Subcn & & M90782 & 001 & 4000 \\
\hline 900004 & K001 & 92734 & Subcn & & M23156 & 001 & 4000 \\
\hline 900005 & L002 & 92734 & Subcn & & M08888 & 001 & 4000 \\
\hline ubtotal & & & & & & & 124000 \\
\hline
\end{tabular}

\section{Material/Contracts (from Passport)}

\begin{tabular}{|c|c|c|c|c|c|c|c|c|c|}
\hline Project ID & $\underline{\mathrm{COA}}$ & Dept ID & Res Type & Res & PO\# & Line & Base \$ & $\operatorname{Tax} \$$ & Total $\$$ \\
\hline 100001 & A001 & 10539 & Mater & & W12345 & 001 & 1000 & 80 & 1080 \\
\hline 300006 & A004 & 10539 & Mater & & W77889 & 001 & 2700 & 216 & 2916 \\
\hline 400002 & D002 & 10539 & Subcn & & M12345 & 001 & 3000 & & 3000 \\
\hline 500002 & N001 & 10539 & Subcn & & M99000 & 001 & 4200 & & 4200 \\
\hline 500004 & A001 & 10539 & Subcn & & M77000 & 001 & 3900 & & 3900 \\
\hline
\end{tabular}


HANDI Fluor Daniel Hanford - PeopleSoft Financials

PAGE 4 of 32

\begin{tabular}{|c|c|c|c|c|c|c|c|c|}
\hline 500013 & N001 & 10539 & Subcn & $M 12321$ & 001 & 8000 & & 8000 \\
\hline 800003 & Do02 & 10539 & Subcn & M15853 & 001 & 11000 & & 11000 \\
\hline 900001 & E003 & 10539 & Subcn & M12673 & 001 & 12000 & & 12000 \\
\hline 900002 & F004 & 10539 & Subcn & M98467 & 001 & 13000 & & 13000 \\
\hline 900004 & K001 & 10539 & Subcn & M07777 & 001 & 1500 & & 1500 \\
\hline 900006 & N001 & 10539 & Subcn & M04444 & 001 & 2100 & & 2100 \\
\hline 900007 & P001 & 10539 & Subcn & M00333 & 001 & 4500 & & 4500 \\
\hline 100002 & $\mathrm{~A} 004$ & 32592 & Mater & W23455 & 001 & 1100 & 88 & 1188 \\
\hline 100003 & A005 & 32592 & Mater & W44620 & 001 & 1200 & 96 & 1296 \\
\hline 500009 & F004 & 32592 & Subcn & $\mathrm{M} 22000$ & 001 & 6000 & & 6000 \\
\hline 500010 & G008 & 32592 & Subcn & M34543 & 001 & 6500 & & 6500 \\
\hline 800001 & A004 & 32592 & Subcn & M00990 & 001 & 9500 & & 9500 \\
\hline 400001 & A005 & 34912 & Mater & W77789 & 001 & 2800 & 224 & 3024 \\
\hline 400003 & E003 & 34912 & Subcn & M23456 & 001 & 3200 & & 3200 \\
\hline 400004 & F004 & 34912 & Subcn & M34567 & 001 & 3400 & & 3400 \\
\hline 400005 & G008 & 34912 & Subcn & M45678 & 001 & 3600 & & 3600 \\
\hline 500011 & K001 & 34912 & Subcn & M56765 & 001 & 7000 & & 7000 \\
\hline 500012 & L.002 & 34912 & Subcn & M89098 & 001 & 7500 & & 7500 \\
\hline 100004 & Do02 & 39754 & Mater & W12334 & 001 & 1300 & 104 & 1404 \\
\hline 300005 & A001 & 39754 & Mater & W00098 & 001 & 800 & 64 & 864 \\
\hline 300005 & $\mathrm{~A} 001$ & 39754 & Mater & W56678 & 001 & 2500 & 200 & 2700 \\
\hline 400006 & K001 & 39754 & Subcn & M56789 & 001 & 3800 & & 3800 \\
\hline 500007 & Do02 & 39754 & Subcn & $M 44000$ & 001 & 3300 & & 3300 \\
\hline 500008 & E003 & 39754 & Subcn & M33000 & 001 & 3100 & & 3100 \\
\hline 300001 & K001. & 42801 & Mater & W56667 & 001 & 1200 & 96 & 1296 \\
\hline 300002 & L002 & 42801 & Mater & W90009 & 001 & 1100 & 88 & 1188 \\
\hline 300003 & N001 & 42801 & Mater & W12230 & 001 & 1000 & 80 & 1080 \\
\hline 300004 & P001 & 42801 & Mater & W23349 & 001 & 900 & 72 & 972 \\
\hline 500014 & P001 & 42801 & Subcn & M67766 & 001 & 8500 & & 8500 \\
\hline 500015 & $\mathrm{~A} 001$ & 42801 & Subcn & M34433 & 001 & 9000 & & 9000 \\
\hline 200001 & E003 & 47610 & Mater & W86788 & 001 & 1500 & 120 & 1620 \\
\hline 200002 & F004 & 47610 & Mater & W24365 & 001 & 1400 & 112 & 1512 \\
\hline 200003 & G008 & 47610 & Mater & W33367 & 001 & 1300 & 104 & 1404 \\
\hline 500006 & $\mathrm{~A} 005$ & 47610 & Subcn & M55000 & 001 & 3500 & & 3500 \\
\hline 100004 & $\mathrm{D} 002$ & 92734 & Mater & W12334 & 002 & 1400 & 112 & 1512 \\
\hline 300006 & A004 & 92734 & Mater & W67899 & 001 & 2600 & 208 & 2808 \\
\hline 500001 & L002 & 92734 & Subcn & M67890 & 001 & 4000 & & 4000 \\
\hline 500003 & P001 & 92734 & Subcn & M88000 & 001 & 4100 & & 4100 \\
\hline 500005 & A004 & 92734 & Subcn & M66000 & 001 & 3700 & & 3700 \\
\hline 800002 & A005 & 92734 & Subcn & M53708 & 001 & 10000 & & 10000 \\
\hline 900003 & G008 & 92734 & Subcn & M90782 & 001 & 900 & & 900 \\
\hline 900004 & K001 & 92734 & Subcn & M23156 & 001 & 1200 & & 1200 \\
\hline 900005 & L002 & 92734 & Subcn & M08888 & 001 & 1800 & & 1800 \\
\hline 900008 & A004 & 92734 & Mater & M08899 & 001 & 1000 & & 1000 \\
\hline subtotal & & & & & & 193600 & 2064 & 195664 \\
\hline
\end{tabular}

6. Labor (from TIS)

\begin{tabular}{|c|c|c|c|c|c|c|c|c|c|}
\hline Project ID & $\mathrm{COA}$ & Dept ID & Res Type & Res Categ & Tot $\$$ & Iot cos & PTB $13 \%$ & Abs $2 \%$ & Total \$ \\
\hline 100001 & $\overline{A 001}$ & 10539 & Labor & $\mathrm{CO00}$ & 3240 & 924 & 312 & 48 & 4524 \\
\hline 100002 & A004 & 32592 & Labor & E000 & 4860 & 1926 & 468 & 72 & 7326 \\
\hline 100003 & A005 & 32592 & Labor & MOOO & 2430 & 963 & 234 & 36 & 3663 \\
\hline 100004 & $\mathrm{DOO} 2$ & 39754 & Labor & E000 & 2430 & 963 & 234 & 36 & 3663 \\
\hline
\end{tabular}


HANDI Fluor Daniel Hanford - PeopleSoft Financials

\begin{tabular}{|c|c|c|c|c|c|c|c|c|c|}
\hline 100004 & $\mathrm{D} 002$ & 92734 & Labor & $E 000$ & 2430 & 963 & 234 & 36 & 3663 \\
\hline 200001 & E003 & 47610 & Labor & $E 000$ & 810 & 321 & 78 & 12 & 1221 \\
\hline 200002 & F004 & 47610 & Labor & M000 & 4050 & 1605 & 390 & 60 & 6105 \\
\hline 200003 & G008 & 47610 & Labor & $E 000$ & 3240 & 1284 & 312 & 48 & 4884 \\
\hline 300001 & K001 & 42801 & Labor & $E 000$ & 8910 & 3531 & 858 & 132 & 13431 \\
\hline 300002 & L002 & 42801 & Labor & M000 & 6075 & 2407.5 & 585 & 90 & 9157.5 \\
\hline 300003 & N001 & 42801 & Labor & M000 & 3240 & 1284 & 312 & 48 & 4884 \\
\hline 300004 & P001 & 42801 & Labor & $E 000$ & 3240 & 1284 & 312 & 48 & 4884 \\
\hline 300005 & A001 & 39754 & Labor & $E 000$ & 4860 & 1926 & 468 & 72 & 7326 \\
\hline 300005 & $\mathrm{~A} 001$ & 39754 & Labor & MO00 & 4860 & 1926 & 468 & 72 & 7326 \\
\hline 300006 & A004 & 92734 & Labor & $E 000$ & 2430 & 963 & 234 & 36 & 3663 \\
\hline 300006 & A004 & 10539 & Labor & $\mathrm{C} 000$ & 2430 & 963 & 234 & 36 & 3663 \\
\hline 400001 & A005 & 34912 & Labor & Mo00 & 2430 & 963 & 234 & 36 & 3663 \\
\hline 400002 & D002 & 10539 & Labor & $\mathrm{C} 000$ & 810 & 321 & 78 & 12 & 1221 \\
\hline 400003 & E003 & 34912 & Labor & $E 000$ & 4050 & 1605 & 390 & 60 & 6105 \\
\hline 400004 & $\mathrm{~F} 004$ & 34912 & Labor & M000 & 3240 & 1284 & 312 & 48 & 4884 \\
\hline 400005 & G008 & 34912 & Labor & $\mathrm{E} 000$ & 8910 & 3531 & 858 & 132 & 13431 \\
\hline 400006 & K001 & 39754 & Labor & E000 & 6075 & 2407.5 & 585 & 90 & 9157.5 \\
\hline 500001 & L002 & 92734 & Labor & M000 & 3240 & 1284 & 312 & 48 & 4884 \\
\hline 500002 & No01 & 10539 & Labor & $\mathrm{CO00}$ & 3240 & 1284 & 312 & 48 & 4884 \\
\hline 500003 & P001 & 92734 & Labor & $E 000$ & 4860 & 1926 & 468 & 72 & 7326 \\
\hline 500004 & A001 & 10539 & Labor & M000 & 2430 & 963 & 234 & 36 & 3663 \\
\hline 500005 & A004 & 92734 & Labor & $E 000$ & 2430 & 963 & 234 & 36 & 3663 \\
\hline 500006 & A005 & 47610 & Labor & MO00 & 810 & 321 & 78 & 12 & 1221 \\
\hline 500007 & D002 & 39754 & Labor & $E 000$ & 4050 & 1605 & 390 & 60 & 6105 \\
\hline 500008 & E003 & 39754 & Labor & $E 000$ & 3240 & 1284 & 312 & 48 & 4884 \\
\hline 500009 & F004 & 32592 & Labor & Mo00 & 8910 & 3531 & 858 & 132 & 13431 \\
\hline 500010 & G008 & 32592 & Labor & Mo00 & 6075 & 2407.5 & 585 & 90 & 9157.5 \\
\hline 500011 & K001 & 34912 & Labor & $E 000$ & 3240 & 1284 & 312 & 48 & 4884 \\
\hline 500012 & L002 & 34912 & Labor & Mo00 & 3645 & 1444.5 & 351 & 54 & 5494.5 \\
\hline 500013 & N001 & 10539 & Labor & $\mathrm{C} 000$ & 3645 & 1444.5 & 351 & 54 & 5494.5 \\
\hline 500014 & P001 & 42801 & Labor & E000 & 8100 & 3210 & 780 & 120 & 12210 \\
\hline 500015 & A001 & 42801 & Labor & E000 & 8100 & 3210 & 780 & 120 & 12210 \\
\hline 800001 & $\mathrm{~A} 004$ & 32592 & Labor & E000 & 3240 & 1284 & 312 & 48 & 4884 \\
\hline 800002 & A005 & 92734 & Labor & MOO0 & 3240 & 1284 & 312 & 48 & 4884 \\
\hline 800003 & D002 & 10539 & Labor & $\mathrm{C} 000$ & 3240 & 1284 & 312 & 48 & 4884 \\
\hline 900001 & E003 & 10539 & Labor & E000 & 5670 & 2247 & 546 & 84 & 8547 \\
\hline 900002 & F004 & 10539 & Labor & MO00 & 6480 & 2568 & 624 & 96 & 9768 \\
\hline 900003 & G008 & 92734 & Labor & E000 & 6885 & 2728.5 & 663 & 102 & 10378.5 \\
\hline 900004 & K001 & 92734 & Labor & E000 & 6480 & 2568 & 624 & 96 & 9768 \\
\hline 900004 & K001 & 10539 & Labor & $\mathrm{C} 000$ & 6480 & 2568 & 624 & 96 & 9768 \\
\hline 900005 & L002 & 92734 & Labor & MO00 & 8100 & 3210 & 780 & 120 & 12210 \\
\hline 900006 & N001 & 10539 & Labor & $\mathrm{COO0}$ & 3240 & 1284 & 312 & 48 & 4884 \\
\hline 900007 & P001 & 10539 & Labor & M000 & 3240 & 1284 & 312 & 48 & 4884 \\
\hline Subtotals & & & & & 207360 & 81816 & 19968 & 3072 & 312216 \\
\hline Info Only & & & & & 20000 & & & & 20000 \\
\hline
\end{tabular}

\section{MPR (from Allocation)}


Rate $=9 \%$

Base $=$ All Project ID costs with resource type $=$ Mater and Subch

Resource Type for allocation $=$ Over

Resource Category for allocation $=$ MPR

Project ID for credit $=900003$

\section{Allocation Methodology for Pool Liquidations}

The purpose of a pool liquidation such as MPR is to add a burden to the base cost for a direct project and reduce the burden amount from the indirect projects (MPR). The transfer of costs between projects needs to be recognized within both General Ledger and Projects.

The MPR allocation process is a two step process. The first step transfers costs between Project IDs within General Ledger account 813260 (MPR). The entry debits 813260 with the direct Project IDs and credits the account with the indirect Projects ID (MPR Project). The basis for the cost transfer was a $9 \%$ additional charge to all projects ID with a resource type of Material or SubContractor. The second step transfers the costs between project IDs within the Projects module.
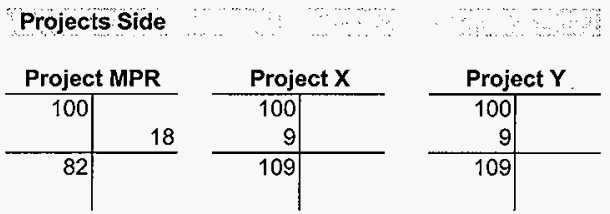

\section{Allocation Methodology for HUB Entries}

The purpose of a HUB entry such as Graphics is to bill the direct projects for using the services of an indirect project (Graphics). The transfer of costs between projects needs to be recognized within both General Ledger and Projects.

The allocation process for HUB entries is a two step process. The first step recognizes the incoming HUB bill in Projects and then debits the General Ledger account 813250 (ODC) with the direct projects ID and credits account 999999 with the direct project IDs. The second allocation recognizes the reduction of costs to the indirect project (Graphics) within Projects and then debits the General Ledger account 999999 with the indirect Project IDs and credits 813250 (ODC) with the indirect Project IDs.
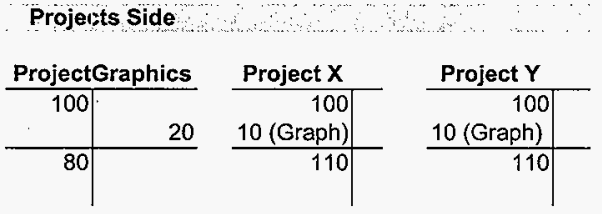

\begin{tabular}{r|rcr|r}
\multicolumn{2}{c}{ 813250- ODC } & \multicolumn{2}{c}{ 999999-Clearing } \\
\hline 10 (proj $X)$ & 20 (proj Graph) & 20 (Graph) & $10($ proj $X)$ \\
10 (proj $Y)$ & & & $10($ proj $Y)$ \\
\hline 0 & & 0 &
\end{tabular}

\section{Data Management Process Flow Diagram for Interfacing Systems}


HANDI Fluor Daniel Hanford - PeopleSoft Financials FIT GAP ANALYSIS 


\section{Business Management Systems \\ DRAFT Process Flow Diagram Interfacing System to PC to GL}

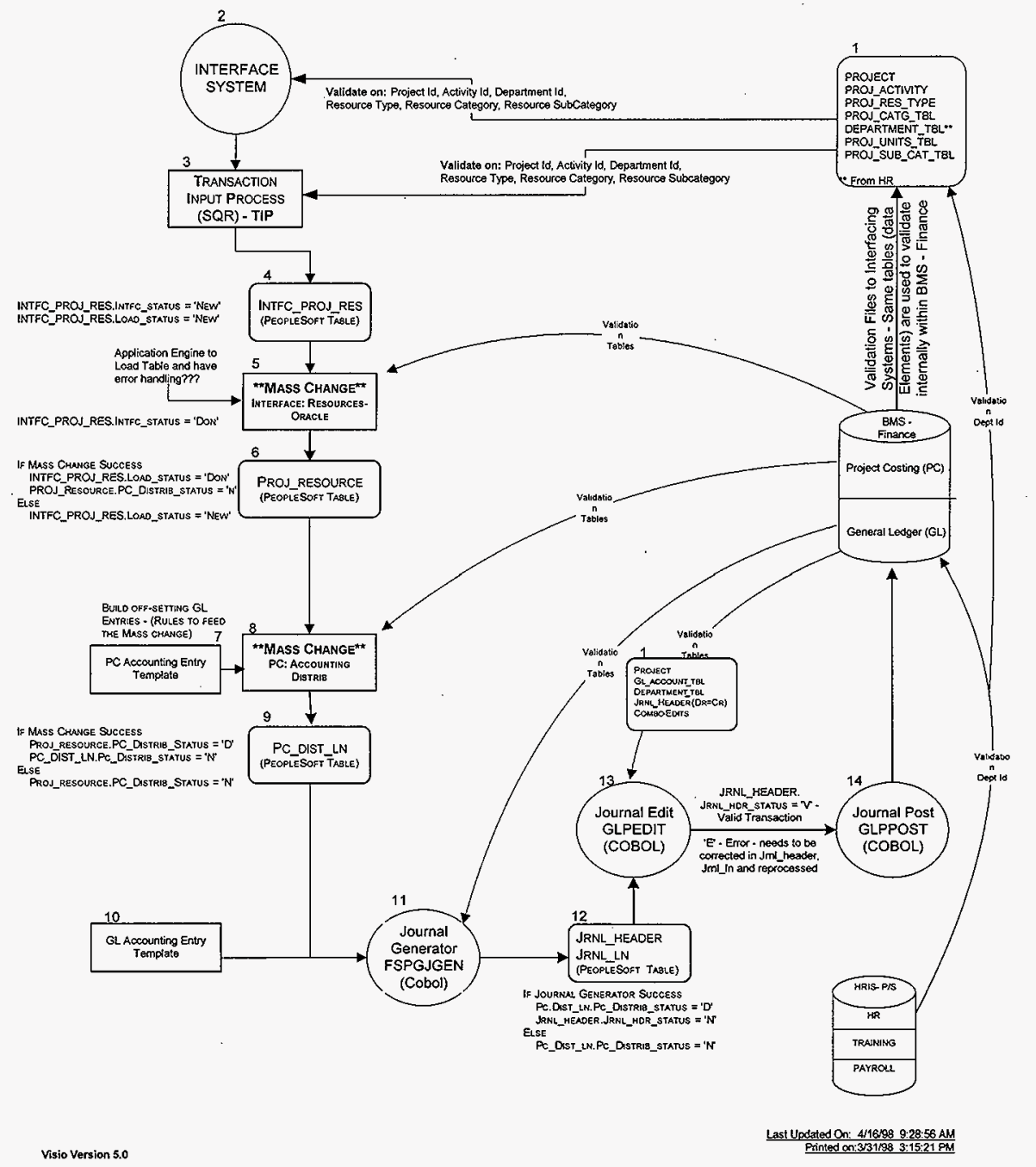


11. FIT/GL Transactional Journal Entries Fit/Gap T-Accounts

\begin{tabular}{|c|c|c|c|c|c|c|}
\hline & & & & & & \\
\hline Account & Journal & Proj & Debit & Credit & Proj & Journal \\
\hline 321200 & 0000005198 & & $44,145.00$ & $-3,240.00$ & 100001 & PC00005206 \\
\hline 321200 & 0000005198 & & $25,515.00$ & $-4,860.00$ & 100002 & PC00005206 \\
\hline 321200 & 0000005198 & & $25,515.00$ & $-2,430.00$ & 100003 & PC00005206 \\
\hline 321200 & 0000005198 & & $25,515.00$ & $-2,430.00$ & 100004 & PC00005206 \\
\hline 321200 & 0000005198 & & $37,665.00$ & $-2,430.00$ & 100004 & PC00005206 \\
\hline 321200 & 0000005198 & & $8,910.00$ & -810.00 & 200001 & $P C 00005206$ \\
\hline 321200 & 0000005198 & & $40,095.00$ & $-4,050.00$ & 200002 & PC00005206 \\
\hline 321200 & & & & $-3,240.00$ & 200003 & PC00005206 \\
\hline 321200 & & & & $-8,910.00$ & 300001 & PC00005206 \\
\hline 321200 & & & & $-6,075.00$ & 300002 & PC00005206 \\
\hline 321200 & & & & $-3,240.00$ & 300003 & PC00005206 \\
\hline 321200 & & & & $-3,240.00$ & 300004 & PC00005206 \\
\hline 321200 & & & & $-9,720.00$ & 300005 & PC00005206 \\
\hline 321200 & & & & $-2,430.00$ & 300006 & PC00005206 \\
\hline 321200 & & & & $-2,430.00$ & 300006 & PC00005206 \\
\hline 321200 & & & & $-2,430.00$ & 400001 & PC00005206 \\
\hline 321200 & & & & -810.00 & 400002 & PC00005206 \\
\hline 321200 & & & & $-4,050.00$ & 400003 & PC00005206 \\
\hline 321200 & & & & $-3,240.00$ & 400004 & PC00005206 \\
\hline 321200 & & & & $-8,910.00$ & 400005 & PC00005206 \\
\hline 321200 & & & & $-6,075.00$ & 400006 & PC00005206 \\
\hline 321200 & & & & $-3,240.00$ & 500001 & $\mathrm{PC} 00005206$ \\
\hline 321200 & & & & $-3,240,00$ & 500002 & PC00005206 \\
\hline 321200 & & & & $-4,860.00$ & 500003 & PC00005206 \\
\hline 321200 & & & & $-2,430.00$ & 500004 & PC00005206 \\
\hline 321200 & & & & $-2,430.00$ & 500005 & $P C 00005206$ \\
\hline 321200 & & & & -810.00 & 500006 & PC00005206 \\
\hline 321200 & & & & $-4,050.00$ & 500007 & PC00005206 \\
\hline 321200 & & & & $-3,240,00$ & 500008 & PC00005206 \\
\hline 321200 & & & & $-8,910.00$ & 500009 & $P C 00005206$ \\
\hline 321200 & & & & $-6,075.00$ & 500010 & $P C 00005206$ \\
\hline 321200 & & & & $-3,240.00$ & 500011 & PC00005206 \\
\hline 321200 & & & & $-3,645.00$ & 500012 & PC00005206 \\
\hline 321200 & & & & $-3,645.00$ & 500013 & PC00005206 \\
\hline 321200 & & & & $-8,100.00$ & 500014 & PC00005206 \\
\hline 321200 & & & & $-8,100.00$ & 500015 & $P C 00005206$ \\
\hline 321200 & & & & $-3,240.00$ & 800001 & $P C 00005206$ \\
\hline 321200 & & & & $-3,240.00$ & 800002 & PC00005206 \\
\hline 321200 & & & & $-3,240.00$ & 800003 & PC00005206 \\
\hline 321200 & & & & $-5,670.00$ & 900001 & PC00005206 \\
\hline 321200 & & & & $-6,480.00$ & 900002 & PC00005206 \\
\hline 321200 & & & & $-6,885.00$ & 900003 & PC00005206 \\
\hline 321200 & & & & $-6,480.00$ & 900004 & PC00005206 \\
\hline
\end{tabular}


HNF-2746 REV 0

PAGE 10 of 32

\begin{tabular}{|c|c|c|c|c|c|c|}
\hline 321200 & & & & $-6,480.00$ & 900004 & PC00005206 \\
\hline 321200 & & & & $-8,100.00$ & 900005 & PC00005206 \\
\hline 321200 & & & & $-3,240.00$ & 900006 & PC00005206 \\
\hline 321200 & & & & $-3,240.00$ & 900007 & PC00005206 \\
\hline & & & $207,360.00$ & $-207,360.00$ & & \\
\hline & & & & & & \\
\hline 321208 & 0000005198 & & 500.00 & -48.00 & 100001 & PC00005206 \\
\hline 321208 & 0000005198 & & 700.00 & -72.00 & 100002 & PC00005206 \\
\hline 321208 & 0000005198 & & 800.00 & -36.00 & 100003 & PC00005206 \\
\hline 321208 & 0000005198 & & $1,000.00$ & -36.00 & 100004 & PC00005206 \\
\hline 321208 & 0000005198 & & 200.00 & -36.00 & 100004 & PC00005206 \\
\hline 321208 & 0000005198 & & 400.00 & -12.00 & 200001 & $\mathrm{PC} 00005206$ \\
\hline 321208 & 0000005198 & & 400.00 & -60.00 & 200002 & PC00005206 \\
\hline 321208 & & & & -48.00 & 200003 & PC00005206 \\
\hline 321208 & & & & -132.00 & 300001 & PC00005206 \\
\hline 321208 & & & & -90.00 & 300002 & PC00005206 \\
\hline 321208 & & & & -48.00 & 300003 & PC00005206 \\
\hline 321208 & & & & -48.00 & 300004 & PC00005206 \\
\hline 321208 & & & & -144.00 & 300005 & PC00005206 \\
\hline 321208 & & & & -36.00 & 300006 & PC00005206 \\
\hline 321208 & & & & -36.00 & 300006 & PC00005206 \\
\hline 321208 & & & & -36.00 & 400001 & PC00005206 \\
\hline 321208 & & & & -12.00 & 400002 & PC00005206 \\
\hline 321208 & & & & -60.00 & 400003 & PC00005206 \\
\hline 321208 & & & & -48.00 & 400004 & PC00005206 \\
\hline 321208 & & & & -132.00 & 400005 & PC00005206 \\
\hline 321208 & & & & -90.00 & 400006 & PC00005206 \\
\hline 321208 & & & & -48.00 & 500001 & PC00005206 \\
\hline 321208 & & & & -48.00 & 500002 & PC00005206 \\
\hline 321208 & & & & -72.00 & 500003 & PC00005206 \\
\hline 321208 & & & & -36.00 & 500004 & PC00005206 \\
\hline 321208 & & & & -36.00 & 500005 & PC00005206 \\
\hline 321208 & & & & -12.00 & 500006 & PC00005206 \\
\hline 321208 & & & & -60.00 & 500007 & PC00005206 \\
\hline 321208 & & & & -48.00 & 500008 & PC00005206 \\
\hline 321208 & & & & -132.00 & 500009 & PC00005206 \\
\hline 321208 & & & & -90.00 & 500010 & $P C 00005206$ \\
\hline 321208 & & & & -48.00 & 500011 & PC00005206 \\
\hline 321208 & & & & -54.00 & 500012 & PC00005206 \\
\hline 321208 & & & & -54.00 & 500013 & PC00005206 \\
\hline 321208 & & & & -120.00 & 500014 & PC00005206 \\
\hline 321208 & & & & -120.00 & 500015 & PC00005206 \\
\hline 321208 & & & & -48.00 & 800001 & PC00005206 \\
\hline 321208 & & & & -48.00 & 800002 & $P C 00005206$ \\
\hline 321208 & & & & -48.00 & 800003 & PC00005206 \\
\hline 321208 & & & & -84.00 & 900001 & $P C 00005206$ \\
\hline 321208 & & & & -96.00 & 900002 & PC00005206 \\
\hline 321208 & & & & -102.00 & 900003 & PC00005206 \\
\hline
\end{tabular}




\begin{tabular}{|c|c|c|c|c|c|c|}
\hline 321208 & & & & -96.00 & 900004 & $P C 00005206$ \\
\hline 321208 & & & & -96.00 & 900004 & PC00005206 \\
\hline 321208 & & & & -120.00 & 900005 & PC00005206 \\
\hline 321208 & & & & -48.00 & 900006 & PC00005206 \\
\hline 321208 & & & & -48.00 & 900007 & PC00005206 \\
\hline & & & $4,000.00$ & $-3,072.00$ & & \\
\hline & & & 928.00 & & & \\
\hline & & & & & & \\
\hline 321209 & 0000005198 & & 800.00 & & & \\
\hline 321209 & 0000005198 & & 200.00 & & & \\
\hline & & & $1,000.00$ & & & \\
\hline & & & & & & \\
\hline 321290 & & & & $-4,000.00$ & 400002 & PCA0005205 \\
\hline 321290 & & & & $-4,000.00$ & 400003 & PCA0005205 \\
\hline 321290 & & & & $-4,000.00$ & 400004 & PCA0005205 \\
\hline 321290 & & & & $-4,000.00$ & 400005 & PCA0005205 \\
\hline 321290 & & & & $-4,000.00$ & 400006 & PCA0005205 \\
\hline 321290 & & & & $-4,000.00$ & 500001 & PCA0005205 \\
\hline 321290 & & & & $-4,000.00$ & 500002 & PCA0005205 \\
\hline 321290 & & & & $-4,000.00$ & 500003 & PCA0005205 \\
\hline 321290 & & & & $-4,000.00$ & 500004 & PCA0005205 \\
\hline 321290 & & & & $-4,000.00$ & 500005 & PCA0005205 \\
\hline 321290 & & & & $-4,000.00$ & 500006 & PCA0005205 \\
\hline 321290 & & & & $-4,000.00$ & 500007 & PCA0005205 \\
\hline 321290 & & & & $-4,000.00$ & 500008 & PCA0005205 \\
\hline 321290 & & & & $-4,000.00$ & 500009 & PCA0005205 \\
\hline 321290 & & & & $-4,000.00$ & 500010 & PCA0005205 \\
\hline 321290 & & & & $-4,000.00$ & 500011 & PCA0005205 \\
\hline 321290 & & & & $-4,000.00$ & 500012 & PCA0005205 \\
\hline 321290 & & & & $-4,000.00$ & 500013 & PCA0005205 \\
\hline 321290 & & & & $-4,000.00$ & 500014 & PCA0005205 \\
\hline 321290 & & & & $-4,000.00$ & 500015 & PCA0005205 \\
\hline 321290 & & & & $-4,000.00$ & 800001 & PCA0005205 \\
\hline 321290 & & & & $-4,000.00$ & 800002 & PCA0005205 \\
\hline 321290 & & & & $-4,000.00$ & 800003 & PCA0005205 \\
\hline 321290 & & & & $-4,000.00$ & 900001 & PCA0005205 \\
\hline 321290 & & & & $-4,000.00$ & 900002 & PCA0005205 \\
\hline 321290 & & & & $-4,000.00$ & 900003 & PCA0005205 \\
\hline 321290 & & & & $-4,000.00$ & 900004 & PCA0005205 \\
\hline 321290 & & & & $-4,000.00$ & 900004 & PCA0005205 \\
\hline 321290 & & & & $-4,000.00$ & 900005 & PCA0005205 \\
\hline 321290 & & & & $-4,000.00$ & 900006 & PCA0005205 \\
\hline 321290 & & & & $-4,000.00$ & 900007 & PCA0005205 \\
\hline & & & & $-124,000.00$ & & \\
\hline & & & & & & \\
\hline 321291 & & & & $-1,080.00$ & 100001 & PC00005206 \\
\hline 321291 & & & & $-1,188.00$ & 100002 & PC00005206 \\
\hline 321291 & & & & $-1,296.00$ & 100003 & PC00005206 \\
\hline
\end{tabular}


HNF-2746 REV 0

PAGE 12 of 32

\begin{tabular}{|c|c|c|c|c|c|c|}
\hline 321291 & & & & $-1,404.00$ & 100004 & PC00005206 \\
\hline 321291 & & & & $-1,512.00$ & 100004 & PC00005206 \\
\hline 321291 & & & & $-1,620.00$ & 200001 & PC00005206 \\
\hline 321291 & & & & $-1,512.00$ & 200002 & PC00005206 \\
\hline 321291 & & & & $-1,404.00$ & 200003 & PC00005206 \\
\hline 321291 & & & & $-1,296.00$ & 300001 & PC00005206 \\
\hline 321291 & & & & $-1,188.00$ & 300002 & PC00005206 \\
\hline 321291 & & & & $-1,080.00$ & 300003 & PC00005206 \\
\hline 321291 & & . & & -972.00 & 300004 & PC00005206 \\
\hline 321291 & & & & $-3,564.00$ & 300005 & PC00005206 \\
\hline 321291 & & & & $-2,808.00$ & 300006 & PC00005206 \\
\hline 321291 & & & & $-2,916.00$ & 300006 & PC00005206 \\
\hline 321291 & & & & $-3,024.00$ & 400001 & PC00005206 \\
\hline 321291 & & & & $-3,000.00$ & 400002 & PC00005206 \\
\hline 321291 & & & & $-3,200.00$ & 400003 & PC00005206 \\
\hline 321291 & & & & $-3,400.00$ & 400004 & PC00005206 \\
\hline 321291 & & & & $-3,600.00$ & 400005 & PC00005206 \\
\hline 321291 & & & & $-3,800.00$ & 400006 & PC00005206 \\
\hline 321291 & & & & $-4,000.00$ & 500001 & PC00005206 \\
\hline 321291 & & & & $-4,200.00$ & 500002 & PC00005206 \\
\hline 321291 & & & & $-4,100.00$ & 500003 & PC00005206 \\
\hline 321291 & & & & $-3,900.00$ & 500004 & PC00005206 \\
\hline 321291 & & & & $-3,700.00$ & 500005 & PC00005206 \\
\hline 321291 & & & & $-3,500.00$ & 500006 & PC00005206 \\
\hline 321291 & & & & $-3,300.00$ & 500007 & PC00005206 \\
\hline 321291 & & & & $-3,100.00$ & 500008 & PC00005206 \\
\hline 321291 & & & & $-6,000.00$ & 500009 & $\mathrm{PC} 00005206$ \\
\hline 321291 & & & & $-6,500.00$ & 500010 & PC00005206 \\
\hline 321291 & & & & $-7,000.00$ & 500011 & PC00005206 \\
\hline 321291 & & & & $-7,500.00$ & 500012 & $\mathrm{PC} 00005206$ \\
\hline 321291 & & & & $-8,000.00$ & 500013 & PC00005206 \\
\hline 321291 & & & & $-8,500.00$ & 500014 & PC00005206 \\
\hline 321291 & & & & $-9,000.00$ & 500015 & PC00005206 \\
\hline 321291 & & & & $-9,500.00$ & 800001 & PC00005206 \\
\hline 321291 & & & & $-10,000.00$ & 800002 & PC00005206 \\
\hline 321291 & & & & $-11,000.00$ & 800003 & PC00005206 \\
\hline 321291 & & & & $-12,000.00$ & 900001 & PC00005206 \\
\hline 321291 & & & & $-13,000.00$ & 900002 & PC00005206 \\
\hline 321291 & & & & -900.00 & 900003 & PC00005206 \\
\hline 321291 & & & & $-1,200.00$ & 900004 & PC00005206 \\
\hline 321291 & & & & $-1,500.00$ & 900004 & PC00005206 \\
\hline 321291 & & & & $-1,800.00$ & 900005 & $P C 00005206$ \\
\hline 321291 & & & & $-2,100.00$ & 900006 & PC00005206 \\
\hline 321291 & & & & $-4,500.00$ & 900007 & PC00005206 \\
\hline 321291 & & & & $-1,000.00$ & 900008 & PC00005206 \\
\hline & & & 0.00 & $-195,664.00$ & & \\
\hline 321299 & & & & -924.00 & 100001 & PC00005206 \\
\hline
\end{tabular}


HNF-2746 REV 0

PAGE 13 of 32

\begin{tabular}{|c|c|c|c|c|c|c|}
\hline 321299 & & & & $-1,926.00$ & 100002 & $\mathrm{PC} 00005206$ \\
\hline 321299 & & & & -963.00 & 100003 & PC00005206 \\
\hline 321299 & & & & -963.00 & 100004 & PC00005206 \\
\hline 321299 & & & & -963.00 & 100004 & PC00005206 \\
\hline 321299 & & & & -321.00 & 200001 & PC00005206 \\
\hline 321299 & & & & $-1,605.00$ & 200002 & PC00005206 \\
\hline 321299 & & & & $-1,284.00$ & 200003 & PC00005206 \\
\hline 321299 & & & & $-3,531.00$ & 300001 & PC00005206 \\
\hline 321299 & & & & $-2,407.50$ & 300002 & PC00005206 \\
\hline 321299 & & & & $-1,284.00$ & 300003 & PC00005206 \\
\hline 321299 & & & & $-1,284.00$ & 300004 & PC00005206 \\
\hline 321299 & & & & $-3,852.00$ & 300005 & PC00005206 \\
\hline 321299 & & & & -963.00 & 300006 & PC00005206 \\
\hline 321299 & & & & -963.00 & 300006 & PC00005206 \\
\hline 321299 & & & & -963.00 & 400001 & PC00005206 \\
\hline 321299 & & & & -321.00 & 400002 & PC00005206 \\
\hline 321299 & & & & $-1,605.00$ & 400003 & PC00005206 \\
\hline 321299 & & & & $-1,284.00$ & 400004 & PC00005206 \\
\hline 321299 & & & & $-3,531.00$ & 400005 & PC00005206 \\
\hline 321299 & & & & $-2,407.50$ & 400006 & PC00005206 \\
\hline 321299 & & & & $-1,284.00$ & 500001 & PC00005206 \\
\hline 321299 & & & & $-1,284.00$ & 500002 & $\mathrm{PC} 00005206$ \\
\hline 321299 & & & & $-1,926.00$ & 500003 & PC00005206 \\
\hline 321299 & & & & -963.00 & 500004 & PC00005206 \\
\hline 321299 & & . & & -963.00 & 500005 & PC00005206 \\
\hline 321299 & & & & -321.00 & 500006 & PC00005206 \\
\hline 321299 & & & & $-1,605.00$ & 500007 & $\mathrm{PC} 00005206$ \\
\hline 321299 & & & & $-1,284.00$ & 500008 & PC00005206 \\
\hline 321299 & & & & $-3,531.00$ & 500009 & PC00005206 \\
\hline 321299 & & & & $-2,407.50$ & 500010 & PC00005206 \\
\hline 321299 & & & & $-1,284.00$ & 500011 & $P C 00005206$ \\
\hline 321299 & & & & $-1,444.50$ & 500012 & PC00005206 \\
\hline 321299 & & & & $-1,444.50$ & 500013 & PC00005206 \\
\hline 321299 & & & & $-3,210.00$ & 500014 & PC00005206 \\
\hline 321299 & & & & $-3,210.00$ & 500015 & PC00005206 \\
\hline 321299 & & & & $-1,284.00$ & 800001 & PC00005206 \\
\hline 321299 & & & & $-1,284.00$ & 800002 & PC00005206 \\
\hline 321299 & & & & $-1,284.00$ & 800003 & PC00005206 \\
\hline 321299 & & & & $-2,247.00$ & 900001 & PC00005206 \\
\hline 321299 & & & & $-2,568.00$ & 900002 & PC00005206 \\
\hline 321299 & & & & $-2,728.50$ & 900003 & $P C 00005206$ \\
\hline 321299 & & & & $-2,568.00$ & 900004 & PC00005206 \\
\hline 321299 & & & & $-2,568.00$ & 900004 & PC00005206 \\
\hline 321299 & & & & $-3,210.00$ & 900005 & PC00005206 \\
\hline 321299 & & & & $-1,284.00$ & 900006 & $\mathrm{PC} 00005206$ \\
\hline 321299 & & & & $-1,284.00$ & 900007 & PC00005206 \\
\hline & & & & $-81,816.00$ & & \\
\hline & & & & & & \\
\hline
\end{tabular}




\begin{tabular}{|c|c|c|c|c|c|c|}
\hline 321400 & 0000005198 & & $1,000.00$ & & & \\
\hline 321400 & 0000005198 & & $1,200.00$ & & & \\
\hline 321400 & 0000005198 & & $2,000.00$ & & & \\
\hline 321400 & 0000005198 & & $4,500.00$ & & & \\
\hline 321400 & 0000005198 & & $2,300.00$ & & & \\
\hline 321400 & 0000005198 & & $3,500.00$ & & & \\
\hline 321400 & 0000005198 & & 500.00 & & & \\
\hline 321400 & & & & -312.00 & 100001 & PC00005206 \\
\hline 321400 & & & & -468.00 & 100002 & PC00005206 \\
\hline 321400 & & & & -234.00 & 100003 & PC00005206 \\
\hline 321400 & & & & -234.00 & 100004 & PC00005206 \\
\hline 321400 & & & & -234.00 & 100004 & PC00005206 \\
\hline 321400 & & & & -78.00 & 200001 & PC00005206 \\
\hline 321400 & & & & -390.00 & 200002 & PC00005206 \\
\hline 321400 & & & & -312.00 & 200003 & $P C 00005206$ \\
\hline 321400 & & & & -858.00 & 300001 & $P C 00005206$ \\
\hline 321400 & & & & -585.00 & 300002 & $P C 00005206$ \\
\hline 321400 & & & & -312.00 & 300003 & PC00005206 \\
\hline 321400 & & & & -312.00 & 300004 & PC00005206 \\
\hline 321400 & & & & -936.00 & 300005 & PC00005206 \\
\hline 321400 & & & & -234.00 & 300006 & PC00005206 \\
\hline 321400 & & & & -234.00 & 300006 & PC00005206 \\
\hline 321400 & & & & -234.00 & 400001 & PC00005206 \\
\hline 321400 & & & & -78.00 & 400002 & PC00005206 \\
\hline 321400 & & & & -390.00 & 400003 & PC00005206 \\
\hline 321400 & & & & -312.00 & 400004 & PC00005206 \\
\hline 321400 & & & & -858.00 & 400005 & PC00005206 \\
\hline 321400 & & & & -585.00 & 400006 & PC00005206 \\
\hline 321400 & & & & -312.00 & 500001 & PC00005206 \\
\hline 321400 & & & & -312.00 & 500002 & PC00005206 \\
\hline 321400 & & & & -468.00 & 500003 & PC00005206 \\
\hline 321400 & & & & -234.00 & 500004 & PC00005206 \\
\hline 321400 & & & & -234.00 & 500005 & $P C 00005206$ \\
\hline 321400 & & & & -78.00 & 500006 & PC00005206 \\
\hline 321400 & & & & -390.00 & 500007 & PC00005206 \\
\hline 321400 & & & & -312.00 & 500008 & PC00005206 \\
\hline 321400 & & & & -858.00 & 500009 & $\mathrm{PC} 00005206$ \\
\hline 321400 & & & & -585.00 & 500010 & PC00005206 \\
\hline 321400 & & & & -312.00 & 500011 & PC00005206 \\
\hline 321400 & & & & -351.00 & 500012 & PC00005206 \\
\hline 321400 & & & & -351.00 & 500013 & PC00005206 \\
\hline 321400 & & & & -780.00 & 500014 & PC00005206 \\
\hline 321400 & & & & -780.00 & 500015 & PC00005206 \\
\hline 321400 & & & & -312.00 & 800001 & $P C 00005206$ \\
\hline 321400 & & & & -312.00 & 800002 & PC00005206 \\
\hline 321400 & & & & -312.00 & 800003 & PC00005206 \\
\hline 321400 & & & & -546.00 & 900001 & PC00005206 \\
\hline 321400 & & & & -624.00 & 900002 & PC00005206 \\
\hline
\end{tabular}




\begin{tabular}{|c|c|c|c|c|c|c|}
\hline 321400 & & & & -663.00 & 900003 & PC00005206 \\
\hline 321400 & & & & -624.00 & 900004 & PC00005206 \\
\hline 321400 & & & & -624.00 & 900004 & PC00005206 \\
\hline 321400 & & & & -780.00 & 900005 & PC00005206 \\
\hline 321400 & & & & -312.00 & 900006 & PC00005206 \\
\hline 321400 & & & & -312.00 & 900007 & PC00005206 \\
\hline & & & $15,000.00$ & $-19,968.00$ & & \\
\hline & & & & $-4,968.00$ & & \\
\hline & & & & & & \\
\hline 321510 & & & & $-12,810.00$ & & 0000005198 \\
\hline 321510 & & & & $-27,415.00$ & & 0000005198 \\
\hline 321510 & & & & $-28,315.00$ & & 0000005198 \\
\hline 321510 & & & & $-31,215.00$ & & 0000005198 \\
\hline 321510 & & & & $-40,165.00$ & & 0000005198 \\
\hline 321510 & & & & $-40,995.00$ & & 0000005198 \\
\hline 321510 & & & & $-46,445.00$ & & 0000005198 \\
\hline & & & & $-227,360.00$ & & \\
\hline & & & & & & \\
\hline 813210 & PC00005206 & 100001 & $4,524.00$ & & & \\
\hline 813210 & PC00005206 & 100002 & $7,326.00$ & & . & \\
\hline 813210 & PC00005206 & 100003 & $3,663.00$ & & & \\
\hline 813210 & PC00005206 & 100004 & $3,663.00$ & & & \\
\hline 813210 & PC00005206 & 100004 & $3,663.00$ & & & \\
\hline 813210 & PC00005206 & 200001 & $1,221.00$ & & & \\
\hline 813210 & PC00005206 & 200002 & $6,105.00$ & & & \\
\hline 813210 & PC00005206 & 200003 & $4,884.00$ & . & & \\
\hline 813210 & PC00005206 & 300001 & $13,431,00$ & & & \\
\hline 813210 & PC00005206 & 300002 & $9,157.50$ & & & \\
\hline 813210 & PC00005206 & 300003 & $4,884,00$ & & & \\
\hline 813210 & PC00005206 & 300004 & $4,884.00$ & & & \\
\hline 813210 & PC00005206 & 300005 & $14,652.00$ & & & \\
\hline 813210 & PC00005206 & 300006 & $3,663.00$ & & & \\
\hline 813210 & PC00005206 & 300006 & $3,663.00$ & & & \\
\hline 813210 & PC00005206 & 400001 & $3,663.00$ & & & \\
\hline 813210 & PC00005206 & 400002 & $1,221.00$ & & & \\
\hline 813210 & PC00005206 & 400003 & $6,105.00$ & & & \\
\hline 813210 & PC00005206 & 400004 & $4,884.00$ & & & \\
\hline 813210 & PC00005206 & 400005 & $13,431.00$ & & & \\
\hline 813210 & PC00005206 & 400006 & $9,157.50$ & & & \\
\hline 813210 & PC00005206 & 500001 & $4,884.00$ & & & \\
\hline 813210 & PC00005206 & 500002 & $4,884.00$ & & & \\
\hline 813210 & PC00005206 & 500003 & $7,326.00$ & & & \\
\hline 813210 & PC00005206 & 500004 & $3,663.00$ & & & \\
\hline 813210 & PC00005206 & 500005 & $3,663.00$ & & & \\
\hline 813210 & PC00005206 & 500006 & $1,221.00$ & & & \\
\hline 813210 & PC00005206 & 500007 & $6,105.00$ & & & \\
\hline 813210 & PC00005206 & 500008 & $4,884.00$ & & & \\
\hline 813210 & PC00005206 & 500009 & $13,431.00$ & & & \\
\hline
\end{tabular}




\begin{tabular}{|c|c|c|c|c|c|c|}
\hline 813210 & PC00005206 & 500010 & $9,157.50$ & & & \\
\hline 813210 & PC00005206 & 500011 & $4,884.00$ & & & \\
\hline 813210 & PC00005206 & 500012 & $5,494.50$ & & & \\
\hline 813210 & PC00005206 & 500013 & $5,494.50$ & & & \\
\hline 813210 & PC00005206 & 500014 & $12,210.00$ & & & \\
\hline 813210 & PC00005206 & 500015 & $12,210.00$ & & & \\
\hline 813210 & PC00005206 & 800001 & $4,884.00$ & & & \\
\hline 813210 & PC00005206 & 800002 & $4,884.00$ & & & \\
\hline 813210 & PC00005206 & 800003 & $4,884.00$ & & & \\
\hline 813210 & PC00005206 & 900001 & $8,547.00$ & & & \\
\hline 813210 & PC00005206 & 900002 & $9,768.00$ & & & \\
\hline 813210 & PC00005206 & 900003 & $10,378.50$ & & & \\
\hline 813210 & PC00005206 & 900004 & $9,768.00$ & & & \\
\hline 813210 & PC00005206 & 900004 & $9,768.00$ & & & \\
\hline 813210 & PC00005206 & 900005 & $12,210.00$ & & & \\
\hline 813210 & PC00005206 & 900006 & $4,884.00$ & & & \\
\hline 813210 & PC00005206 & 900007 & $4,884.00$ & & & \\
\hline & & & $312,216.00$ & & & \\
\hline & & & & & & \\
\hline 813220 & PC00005206 & 100001 & $1,080.00$ & & & \\
\hline 813220 & PC00005206 & 100002 & $1,188.00$ & & & \\
\hline 813220 & PC00005206 & 100003 & $1,296.00$ & & & \\
\hline 813220 & PC00005206 & 100004 & $1,512.00$ & & & \\
\hline 813220 & PC00005206 & 100004 & $1,404.00$ & & & \\
\hline 813220 & PC00005206 & 200001 & $1,620.00$ & & & \\
\hline 813220 & PC00005206 & 200002 & $1,512.00$ & & & \\
\hline 813220 & PC00005206 & 200003 & $1,404.00$ & & & \\
\hline 813220 & PC00005206 & 300001 & $1,296.00$ & & & \\
\hline 813220 & PC00005206 & 300002 & $1,188.00$ & & & \\
\hline 813220 & PC00005206 & 300003 & $1,080.00$ & & & \\
\hline 813220 & PC00005206 & 300004 & 972.00 & & & \\
\hline 813220 & PC00005206 & 300005 & $3,564.00$ & & & \\
\hline 813220 & PC00005206 & 300006 & $2,916.00$ & & & \\
\hline 813220 & PC00005206 & 300006 & $2,808.00$ & & & \\
\hline 813220 & PC00005206 & 400001 & $3,024.00$ & & & \\
\hline 813220 & PC00005206 & 900008 & $1,000.00$ & & & \\
\hline & & & $28,864.00$ & & & \\
\hline & & & & & & \\
\hline 813230 & PCA 0005205 & 500015 & $4,000.00$ & & & \\
\hline 813230 & PCA0005205 & 800001 & $4,000.00$ & & & \\
\hline 813230 & PCA0005205 & 800002 & $4,000.00$ & & & \\
\hline 813230 & PCA0005205 & 800003 & $4,000.00$ & & & \\
\hline 813230 & PCA0005205 & 900001 & $4,000.00$ & & & \\
\hline 813230 & PCA0005205 & 900002 & $4,000.00$ & & & \\
\hline 813230 & PCA0005205 & 900003 & $4,000.00$ & & & \\
\hline 813230 & PCA0005205 & 900004 & $4,000.00$ & & & \\
\hline 813230 & PCA0005205 & 900004 & $4,000.00$ & & & \\
\hline 813230 & PCA0005205 & 900005 & $4,000.00$ & & & \\
\hline
\end{tabular}




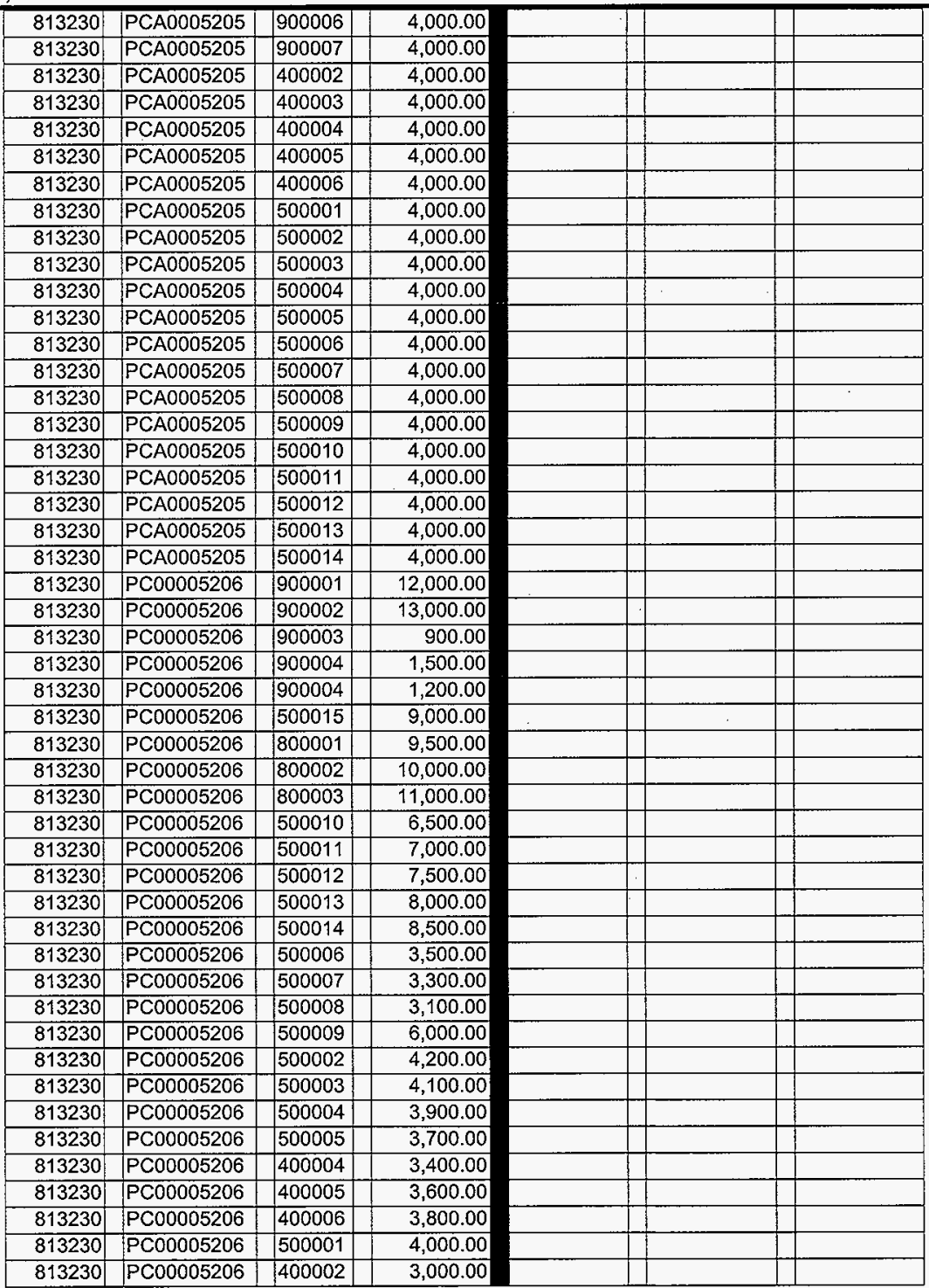


HANDI Fluor Daniel Hanford - PeopleSoft Financials

FIT GAP ANALYSIS

HNF-2746 REV 0

April 17, 1998

PAGE 18 of 32

\begin{tabular}{|c|c|c|c|c|c|c|}
\hline 813230 & PC00005206 & 400003 & $3,200.00$ & & & \\
\hline 813230 & PC00005206 & 900005 & $1,800.00$ & & & \\
\hline 813230 & PC00005206 & 900006 & $2,100.00$ & & & \\
\hline 813230 & PC00005206 & 900007 & $4,500.00$ & & & \\
\hline & & & $290,800.00$ & & & \\
\hline & & & & & & \\
\hline & & & & & & \\
\hline 813250 & PC00005206 & 500001 & $12,500.00$ & $31,000.00$ & 900005 & \\
\hline 813250 & PC00005206 & 900001 & $8,000.00$ & $48,300,00$ & 900006 & \\
\hline 813250 & PC00005206 & 900004 & $8,000.00$ & $27,000.00$ & 900007 & \\
\hline 813250 & PC00005206 & 800001 & $7,300.00$ & & & \\
\hline 813250 & PC00005206 & 900006 & $5,000.00$ & & & \\
\hline 813250 & PC00005206 & 400006 & $4,500.00$ & & & \\
\hline 813250 & PC00005206 & 200001 & $4,500.00$ & & . & \\
\hline 813250 & PC00005206 & 500002 & $4,000.00$ & & & \\
\hline 813250 & PC00005206 & 400005 & $4,000.00$ & & & \\
\hline 813250 & PC00005206 & 900002 & $3,800.00$ & & & \\
\hline 813250 & PC00005206 & 800002 & $3,600.00$ & & & \\
\hline 813250 & PC00005206 & 900003 & $3,500.00$ & & & \\
\hline 813250 & PC00005206 & 400004 & $3,400.00$ & & & \\
\hline 813250 & PC00005206 & 800003 & $3,000.00$ & & & \\
\hline 813250 & $P C 00005206$ & 100004 & $3,000.00$ & & & \\
\hline 813250 & PC00005206 & 300001 & $2,800.00$ & & & \\
\hline 813250 & PC00005206 & 400003 & $2,400.00$ & & & \\
\hline 813250 & PC00005206 & 500010 & $2,300.00$ & & & \\
\hline 813250 & PC00005206 & 500008 & $2,000.00$ & & & \\
\hline 813250 & PC00005206 & 200002 & $2,000.00$ & & & \\
\hline 813250 & PC00005206 & 100003 & $2,000.00$ & & & \\
\hline 813250 & PC00005206 & 200003 & $1,900.00$ & & & \\
\hline 813250 & PC00005206 & 500003 & $1,700.00$ & & & \\
\hline 813250 & PC00005206 & 500015 & $1,500.00$ & & & \\
\hline 813250 & PC00005206 & 100004 & $1,500.00$ & & & \\
\hline 813250 & PC00005206 & 100001 & $1,500.00$ & & & \\
\hline 813250 & PC00005206 & 400002 & $1,400.00$ & & & \\
\hline 813250 & PC00005206 & 300004 & $1,300.00$ & & & \\
\hline 813250 & PC00005206 & 500007 & $1,100.00$ & & & \\
\hline 813250 & PC00005206 & 500006 & $1,000.00$ & & & \\
\hline 813250 & PC00005206 & 500004 & 900.00 & & & \\
\hline 813250 & PC00005206 & 500005 & 600.00 & & & \\
\hline 813250 & $\mathrm{PC} 00005206$ & 500011 & 200.00 & & & \\
\hline 813250 & PC00005206 & 300003 & 100.00 & & & \\
\hline & & & $106,300.00$ & $106,300.00$ & & \\
\hline & & & & & & \\
\hline 813260 & ALO0000001 & 100001 & 97.20 & $-28,769.76$ & 900003 & ALO0000001 \\
\hline 813260 & ALO0000001 & 100002 & 106.92 & & & \\
\hline 813260 & ALO0000001 & 100003 & 116.64 & & & \\
\hline 813260 & ALO0000001 & 100004 & 126.36 & & & \\
\hline 813260 & ALO0000001 & 100004 & 136.08 & & & \\
\hline
\end{tabular}




\begin{tabular}{|c|c|c|c|c|c|c|}
\hline 813260 & ALO0000001 & 200001 & 145.80 & & & \\
\hline 813260 & ALO0000001 & 200002 & 136.08 & & & 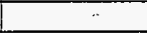 \\
\hline 813260 & ALO0000001 & 200003 & 126.36 & & & \\
\hline 813260 & ALO0000001 & 300001 & 116.64 & & & \\
\hline 813260 & ALO0000001 & 300002 & 106.92 & & & \\
\hline 813260 & ALO0000001 & 300003 & 97.20 & & & \\
\hline 813260 & ALO0000001 & 300004 & 87.48 & & & \\
\hline 813260 & ALO0000001 & 300005 & 320.76 & & & \\
\hline 813260 & ALO0000001 & 300006 & 252.72 & & & \\
\hline 813260 & ALO0000001 & 300006 & 262.44 & & & . \\
\hline 813260 & ALO0000001 & 400001 & 272.16 & & & \\
\hline 813260 & ALO0000001 & 400002 & 630.00 & & & \\
\hline 813260 & ALO0000001 & 400003 & 648.00 & & & \\
\hline 813260 & ALO0000001 & 400004 & 666.00 & & & \\
\hline 813260 & ALO0000001 & 400005 & 684.00 & & & \\
\hline 813260 & ALO0000001 & 400006 & 702.00 & & & \\
\hline 813260 & ALO0000001 & 500001 & 720.00 & & & \\
\hline 813260 & ALO0000001 & 500002 & 738.00 & & & \\
\hline 813260 & ALO0000001 & 500003 & 729.00 & & & \\
\hline 813260 & ALO0000001 & 500004 & 711.00 & & & \\
\hline 813260 & ALO0000001 & 500005 & 693.00 & & & \\
\hline 813260 & ALO0000001 & 500006 & 675.00 & & & \\
\hline 813260 & ALO0000001 & 500007 & 657.00 & & & \\
\hline 813260 & ALO0000001 & 500008 & 639.00 & & & \\
\hline 813260 & ALO0000001 & 500009 & 900.00 & & & \\
\hline 813260 & ALO0000001 & 500010 & 945.00 & & & \\
\hline 813260 & ALO0000001 & 500011 & 990.00 & & & \\
\hline 813260 & ALO0000001 & 500012 & $1,035.00$ & & & \\
\hline 813260 & ALO0000001 & 500013 & $1,080.00$ & & & \\
\hline 813260 & ALO0000001 & 500014 & $1,125.00$ & & & \\
\hline 813260 & ALO0000001 & 500015 & $1,170.00$ & & & \\
\hline 813260 & ALO0000001 & 800001 & $1,215.00$ & & & \\
\hline 813260 & ALO0000001 & 800002 & $1,260.00$ & & & \\
\hline 813260 & ALO0000001 & 800003 & $1,350.00$ & & & \\
\hline 813260 & ALO0000001 & 900001 & $1,440.00$ & . & & \\
\hline 813260 & ALO0000001 & 900002 & $1,530.00$ & & & \\
\hline 813260 & ALO0000001 & 900003 & 441.00 & & & \\
\hline 813260 & ALO0000001 & 900004 & 468.00 & & & \\
\hline 813260 & ALO0000001 & 900004 & 495.00 & & & \\
\hline 813260 & ALO0000001 & 900005 & 522.00 & & & \\
\hline 813260 & ALO0000001 & 900006 & 549.00 & & & \\
\hline 813260 & ALO0000001 & 900007 & 765.00 & & & \\
\hline \multirow[t]{3}{*}{813260} & ALO0000001 & 900008 & 90.00 & & & \\
\hline & & & $28,769.76$ & $-28,769.76$ & & \\
\hline & & & 0.00 & & & \\
\hline & & & & & & \\
\hline 999999 & PC00005207 & 900005 & $31,000.00$ & $-1,500.00$ & 100001 & PC00005206 \\
\hline 999999 & PC00005207 & 900006 & $48,300.00$ & $-2,000.00$ & 100003 & PC00005206 \\
\hline
\end{tabular}


HNF-2746 REV 0

PAGE 20 of 32

\begin{tabular}{|c|c|c|c|c|c|c|}
\hline 999999 & PC00005207 & 900007 & $27,000.00$ & $-1,500.00$ & 100004 & PC00005206 \\
\hline 999999 & & & & $-3,000.00$ & 100004 & PC00005206 \\
\hline 999999 & & & & $-4,500.00$ & 200001 & PC00005206 \\
\hline 999999 & & & & $-2,000.00$ & 200002 & PC00005206 \\
\hline 999999 & & & & $-1,900.00$ & 200003 & PC00005206 \\
\hline 999999 & & & & $-2,800.00$ & 300001 & PC00005206 \\
\hline 999999 & & & & -100.00 & 300003 & PC00005206 \\
\hline 999999 & & & & $-1,300.00$ & 300004 & PC00005206 \\
\hline 999999 & & & & $-1,400.00$ & 400002 & PC00005206 \\
\hline 999999 & & & & $-2,400.00$ & 400003 & PC00005206 \\
\hline 999999 & & & & $-3,400.00$ & 400004 & PC00005206 \\
\hline 999999 & & & & $-4,000.00$ & 400005 & PC00005206 \\
\hline 999999 & & & & $-4,500.00$ & 400006 & PC00005206 \\
\hline 999999 & & & & $-12,500.00$ & 500001 & PC00005206 \\
\hline 999999 & & & & $-4,000.00$ & 500002 & PC00005206 \\
\hline 999999 & & & & $-1,700.00$ & 500003 & PC00005206 \\
\hline 999999 & & & & -900.00 & 500004 & PC00005206 \\
\hline 999999 & & & & -600.00 & 500005 & PC00005206 \\
\hline 999999 & & & & $-1,000.00$ & 500006 & PC00005206 \\
\hline 999999 & & & & $-1,100.00$ & 500007 & $\mathrm{PC} 00005206$ \\
\hline 999999 & & & & $-2,000.00$ & 500008 & PC00005206 \\
\hline 999999 & & & & $-2,300.00$ & 500010 & $\mathrm{PC} 00005206$ \\
\hline 999999 & & & & -200.00 & 500011 & PC00005206 \\
\hline 999999 & & & & $-1,500.00$ & 500015 & PC00005206 \\
\hline 999999 & & & & $-7,300.00$ & 800001 & PC00005206 \\
\hline 999999 & & & & $-3,600.00$ & 800002 & PC00005206 \\
\hline 999999 & & & & $-3,000.00$ & 800003 & PC00005206 \\
\hline 999999 & & & & $-8,000.00$ & 900001 & PC00005206 \\
\hline 999999 & & & & $-3,800.00$ & 900002 & PC00005206 \\
\hline 999999 & & & & $-3,500.00$ & 900003 & PC00005206 \\
\hline 999999 & & & & $-4,000.00$ & 900004 & PC00005206 \\
\hline 999999 & & & & $-4,000.00$ & 900004 & PC00005206 \\
\hline \multirow[t]{2}{*}{999999} & & & & $-5,000.00$ & 900006 & PC00005206 \\
\hline & & & $106,300.00$ & $-106,300.00$ & & \\
\hline
\end{tabular}

\section{FIT/GAP Transactional Project Entries}

\begin{tabular}{|c|l|l|l|l|l|l|l|r|}
\hline Proj & Activity & ResourcelD & An Type & Res Type & Category & Amount \\
\hline 100001 & A001 & 10000013 & ACT & DIRCT & GRAPH & $1,000.00$ \\
\hline 100001 & A001 & 10000016 & ACT & DIRCT & SLC & 500.00 \\
\hline 100001 & A001 & 10000056 & ACT & MATER & & $1,080.00$ \\
\hline 100001 & A001 & 10000066 & ACT & LABOR & C000 & $3,240.00$ \\
\hline 100001 & A001 & 10000074 & ACT & LABOR & COS & 924.00 \\
\hline 100001 & A001 & 10000075 & ACT & LABOR & PTB & 312.00 \\
\hline 100001 & A001 & 10000076 & ACT & LABOR & ABS & 48.00 \\
\hline 100001 & LIQD & PHO00001 & ACT & OVER & MPR & 97.20 \\
\hline
\end{tabular}


PAGE 21 of 32

\begin{tabular}{|c|l|l|l|l|}
\hline Proj & Activity & ResourcelD & \\
\hline 100002 & A004 & 10000070 & \\
\hline 100002 & A004 & 10000100 & \\
\hline 100002 & A004 & 10000101 & \\
\hline 100002 & A004 & 10000102 & \\
\hline 100002 & A004 & 10000103 & \\
\hline 100002 & LIQD & PH000002 & AC \\
\hline
\end{tabular}

\begin{tabular}{|l|l|l|l|}
\hline An Type & Res Type & Category \\
\hline ACT & & MATER & \\
\hline ACT & LABOR & EO00 \\
\hline ACT & LABOR & COS \\
\hline ACT & LABOR & PTB \\
\hline ACT & LABOR & ABS \\
\hline ACT & OVER & MPR \\
\hline
\end{tabular}

Amount

\begin{tabular}{|c|l|l|l|l|l|l|l|r|}
\hline Proj & Activity & ResourcelD & An Type & Res Type & Category & Amount \\
& & & & & & & \\
\hline 100003 & A005 & 10000018 & ACT & DIRCT & SLC & $2,000.00$ \\
\hline 100003 & A005 & 10000071 & ACT & MATER & & $1,296.00$ \\
\hline 100003 & A005 & 10000113 & ACT & LABOR & M000 & $2,430.00$ \\
\hline 100003 & A005 & 10000114 & ACT & LABOR & COS & 963.00 \\
\hline 100003 & A005 & 10000115 & ACT & LABOR & PTB & 234.00 \\
\hline 100003 & AO05 & 10000116 & ACT & LABOR & ABS & 36.00 \\
\hline 100003 & LIQD & PH000003 & ACT & OVER & MPR & 116.64 \\
\hline
\end{tabular}

\begin{tabular}{|c|c|c|c|c|c|c|}
\hline & & & & & & $7,075.64$ \\
\hline Proj & Activity & ResourcelD & An Type & Res Type & Category & Amount \\
\hline 100004 & $\mathrm{D} 002$ & 10000020 & ACT & DIRCT & SLC & $1,500.00$ \\
\hline 100004 & D002 & 10000022 & ACT & DIRCT & SLC & $3,000.00$ \\
\hline 100004 & $\mathrm{D} 002$ & 10000108 & ACT & MATER & & $1,404.00$ \\
\hline 100004 & D002 & 10000136 & ACT & LABOR & $E 000$ & $2,430.00$ \\
\hline 100004 & D002 & 10000137 & ACT & LABOR & $\cos$ & 963.00 \\
\hline 100004 & D002 & 10000138 & ACT & LABOR & PTB & 234.00 \\
\hline 100004 & Do02 & 10000139 & $\overline{A C T}$ & LABOR & ABS & 36.00 \\
\hline 100004 & D002 & 10000147 & ACT & LABOR & E000 & $2,430.00$ \\
\hline 100004 & D002 & 10000148 & ACT & LABOR & $\cos$ & 963.00 \\
\hline 100004 & $\mathrm{D} 002$ & 10000149 & ACT & LABOR & PTB & 234.00 \\
\hline 100004 & $\mathrm{D} 002$ & 10000150 & ACT & LABOR & ABS & 36.00 \\
\hline 100004 & $\mathrm{D} 002$ & 10000164 & ACT & MATER & & $1,512.00$ \\
\hline 100004 & LIQD & $\mathrm{PH} 000004$ & $A C T$ & OVER & MPR & 126.36 \\
\hline 100004 & LIQD & $\mathrm{PH} 000005$ & ACT & OVER & MPR & 136.08 \\
\hline & & & & & & $15,004.44$ \\
\hline Proj & Activity & ResourcelD & An Type & Res Type & Category & Amount \\
\hline 200001 & E003 & 10000024 & $\overline{A C T}$ & DIRCT & SLC & $4,500.00$ \\
\hline 200001 & E003 & 10000155 & ACT & MATER & & $1,620.00$ \\
\hline 200001 & E003 & 10000158 & ACT & LABOR & E000 & 810.00 \\
\hline 200001 & E003 & 10000159 & ACT & LABOR & $\cos$ & 321.00 \\
\hline 200001 & $E 003$ & 10000160 & $\overline{A C T}$ & LABOR & PTB & 78.00 \\
\hline 200001 & $E 003$ & 10000161 & ACT & LABOR & ABS & 12.00 \\
\hline 200001 & LIQD & PH000006 & ACT & OVER & MPR & 145.80 \\
\hline
\end{tabular}


PAGE 22 of 32

\begin{tabular}{|c|l|l|l|}
\hline Proj & Activity & \\
\hline 200002 & F004 & 100 \\
\hline 200002 & F004 & 100 \\
\hline 200002 & F004 & 100 \\
\hline 200002 & F004 & 100 \\
\hline 200002 & F004 & 100 \\
\hline 200002 & F004 & 100 \\
\hline 200002 & LIQD & PH \\
\hline
\end{tabular}

\begin{tabular}{|l|l|l|}
\hline \multicolumn{1}{|c|}{ ResourcelD } & \\
\hline 10000026 & & \\
\hline 10000157 & \\
\hline 10000186 & $A C$ \\
\hline 10000191 & \\
\hline 10000192 & \\
\hline 10000193 & & \\
\hline PH000007 & AC \\
\hline
\end{tabular}

\begin{tabular}{|l|l|l|}
\hline An Type & Res Type \\
\hline ACT & & \\
\hline ACT & DIRCT & \\
\hline ACT & MATER & \\
\hline ACT & LABOR & LABOR \\
\hline ACT & LABOR \\
\hline ACT & LABOR \\
\hline ACT & OVER \\
\hline
\end{tabular}

Category

SLC

\begin{tabular}{|l|l|}
\hline M000 & $1,512.00$ \\
\hline
\end{tabular}

\begin{tabular}{|l|l|}
\hline $\operatorname{COS}$ & $1,605.00$ \\
\hline
\end{tabular}

PTB

ABS

MPR

Amount

\begin{tabular}{|c|l|l|l|}
\hline Proj & Activity & \\
\hline 200003 & G008 & $:$ & \\
\hline 200003 & G008 & & \\
\hline 200003 & G008 & \\
\hline 200003 & G008 & \\
\hline 200003 & G008 & \\
\hline 200003 & G008 & \\
\hline 200003 & G008 & 100 \\
\hline 200003 & LIQD & PH \\
\hline
\end{tabular}

\begin{tabular}{|l|l|}
\hline ResourcelD \\
\hline 10000015 \\
\hline 10000027 \\
\hline 10000162 \\
\hline 10000198 \\
\hline 10000199 \\
\hline 10000200 \\
\hline 10000201 \\
\hline PH000008 \\
\hline
\end{tabular}

\begin{tabular}{|l|l|l}
\hline An Type & Res \\
\hline ACT & DIR \\
\hline ACT & DIR \\
\hline ACT & NCAT \\
\hline ACT & LAB \\
\hline ACT & LAB \\
\hline ACT & LAB \\
\hline ACT & LAB \\
\hline ACT & O \\
\hline
\end{tabular}

\begin{tabular}{|l|l|l}
\hline Res Type & Category \\
& \\
\hline DIRCT & GRAPH \\
\hline DIRCT & SLC \\
\hline MATER & \\
\hline LABOR & EOOO \\
\hline LABOR & COS \\
\hline LABOR & PTB \\
\hline LABOR & ABS \\
\hline OVER & MPR \\
\hline
\end{tabular}

$9,753.08$

\begin{tabular}{|l|l|l|l|l|}
\hline Proj & Activity & ResourcelD & An \\
& & & & \\
\hline 300001 & K001 & 10000017 & ACT \\
\hline 300001 & K001 & 10000029 & ACT \\
\hline 300001 & K001 & 10000130 & AC \\
\hline 300001 & K001 & 10000210 & ACT \\
\hline 300001 & K001 & 10000211 & ACT \\
\hline 300001 & K001 & 10000212 & ACT \\
\hline 300001 & K001 & 10000221 & ACT \\
\hline 300001 & LIQD & PH000009 & ACT \\
\hline
\end{tabular}

\begin{tabular}{|l|l|l}
\hline An Type & R \\
\hline ACT & D \\
\hline ACT & DIR \\
\hline ACT & NAT \\
\hline ACT & LAB \\
\hline ACT & LAB \\
\hline ACT & LAB \\
\hline ACT & LAB \\
\hline ACT & OCVE \\
\hline
\end{tabular}

\begin{tabular}{|l|l|}
\hline Res Typ \\
\hline DIRCT \\
\hline DIRCT \\
\hline MATER \\
\hline LABOR \\
\hline LABOR \\
\hline LABOR \\
\hline LABOR \\
\hline OVER \\
\hline
\end{tabular}

\begin{tabular}{|l|l|}
\hline Category \\
\hline GRAPH \\
\hline SLC \\
\hline & \\
\hline EOOO & \\
\hline COS & \\
\hline ABS & PTB \\
\hline MPR & \\
\hline
\end{tabular}

$8,314.36$

\begin{tabular}{|c|l|l|l|l|l|}
\hline Proj & Activity & ResourceID & An \\
& & & & \\
\hline 300002 & LO02 & 10000135 & ACT \\
\hline 300002 & LOO2 & 10000213 & ACT \\
\hline 300002 & LO02 & 10000214 & ACT \\
\hline 300002 & LO02 & 10000215 & ACT \\
\hline 300002 & LO02 & 10000216 & ACT \\
\hline 300002 & LIQD & PH000010 & ACT \\
\hline
\end{tabular}

\begin{tabular}{|c|l|l|l|l|l|l|l|l|r|}
\hline Proj & Activity & & ResourcelD & An Type & Res Type & Category & Amount \\
\hline 300003 & LIQD & PH000011 & ACT & OVER & MPR & 97.20 \\
\hline 300003 & N001 & 10000030 & ACT & DIRCT & SLC & 100.00 \\
\hline 300003 & N001 & 10000140 & ACT & MATER & & & $1,080.00$ \\
\hline 300003 & N001 & 10000246 & ACT & LABOR & M000 & $3,240.00$ \\
\hline
\end{tabular}


HANDI Fluor Daniel Hanford - PeopleSoft Financials

FIT GAP ANALYSIS

HNF-2746 REV 0

April 17, 1998

PAGE 23 of 32

\begin{tabular}{|c|c|c|c|c|c|c|}
\hline 300003 & N001 & 10000247 & $A C T$ & LABOR & Cos & $1,284.00$ \\
\hline 300003 & N001 & 10000248 & ACT & LABOR & PTB & 312.00 \\
\hline 300003 & N001 & 10000249 & ACT & LABOR & ABS & 48.00 \\
\hline & & & & & & $6,161.20$ \\
\hline Proj & Activity & ResourcelD & An Type & Res Type & Category & Amount \\
\hline 300004 & LIQD & $\mathrm{PH} 000012$ & ACT & OVER & MPR & 87.48 \\
\hline 300004 & P001 & 10000054 & ACT & DIRCT & $S L C$ & $1,300.00$ \\
\hline 300004 & P001 & 10000145 & ACT & MATER & & 972.00 \\
\hline 300004 & P001 & 10000258 & ACT & LABOR & $E 000$ & $3,240.00$ \\
\hline 300004 & P001 & 10000260 & ACT & LABOR & $\cos$ & $1,284.00$ \\
\hline 300004 & P001 & 10000261 & ACT & LABOR & PTB & 312.00 \\
\hline 300004 & P001 & 10000262 & ACT & LABOR & ABS & 48.00 \\
\hline & & & & & & $7,243.48$ \\
\hline Proj & Activity & ResourcelD & An Type & Res Type & Category & Amount \\
\hline 300005 & $A 001$ & 10000109 & ACT & MATER & & 864.00 \\
\hline 300005 & A001 & 10000118 & $\mathrm{ACT}$ & MATER & & $2,700.00$ \\
\hline 300005 & A001 & 10000271 & ACT & LABOR & E000 & $4,860.00$ \\
\hline 300005 & A001 & 10000272 & ACT & LABOR & $\cos$ & $1,926.00$ \\
\hline 300005 & $A 001$ & 10000273 & ACT & LABOR & PTB & 468.00 \\
\hline 300005 & A001 & 10000274 & ACT & LABOR & ABS & 72.00 \\
\hline 300005 & A001 & 10000279 & ACT & LABOR & M000 & $4,860.00$ \\
\hline 300005 & $A 001$ & 10000280 & ACT & LABOR & COS & $1,926.00$ \\
\hline 300005 & $\overline{A 001}$ & 10000281 & ACT & LABOR & PTB & 468.00 \\
\hline 300005 & $A 001$ & 10000282 & ACT & LABOR & ABS & 72.00 \\
\hline 300005 & LIQD & $\mathrm{PH} 000013$ & ACT & OVER & MPR & 320.76 \\
\hline & & & & & & $18,536.76$ \\
\hline Proj & Activity & ResourcelD & An Type & Res Type & Category & Amount \\
\hline 300006 & $A 004$ & 10000057 & ACT & MATER & & $2,916.00$ \\
\hline 300006 & $A 004$ & 10000165 & ACT & MATER & & $2,808.00$ \\
\hline 300006 & $\mathrm{~A} 004$ & 10000283 & ACT & LABOR & E000 & $2,430.00$ \\
\hline 300006 & A004 & 10000284 & ACT & LABOR & $\cos$ & 963.00 \\
\hline 300006 & $\mathrm{~A} 004$ & 10000285 & ACT & LABOR & PTB & 234.00 \\
\hline 300006 & A004 & 10000286 & ACT & LABOR & $A B S$ & 36.00 \\
\hline 300006 & A004 & 10000287 & ACT & LABOR & $C 000$ & $2,430.00$ \\
\hline 300006 & A004 & 10000288 & ACT & LABOR & $\cos$ & 963.00 \\
\hline 300006 & A004 & 10000289 & ACT & LABOR & PTB & 234.00 \\
\hline 300006 & A004 & 10000290 & ACT & LABOR & ABS & 36.00 \\
\hline 300006 & LIQD & $\mathrm{PH} 000014$ & ACT & OVER & MPR & 262.44 \\
\hline 300006 & LIQD & $\mathrm{PH} 000015$ & ACT & OVER & MPR & 252.72 \\
\hline & & & & & & $13,565.16$ \\
\hline Proj & Activity & ResourcelD & An Type & Res Type & Category & Amount \\
\hline 400001 & A005 & 10000088 & ACT & MATER & & $3,024.00$ \\
\hline 400001 & A005 & 10000291 & ACT & LABOR & M000 & $2,430.00$ \\
\hline
\end{tabular}




\begin{tabular}{|l|l|l|l|l|}
\hline 400001 & A005 & 10000292 & & AC \\
\hline 400001 & A005 & 10000293 & & AC \\
\hline 400001 & A005 & 10000294 & & AC \\
\hline 400001 & LIQD & PH000016 & AC \\
\hline
\end{tabular}

\begin{tabular}{|c|l|l|l|l|l|l|l|l|l|}
\hline Proj & Activity & ResourcelD & An Type & Res Type & Category & \\
& & & & & & & \\
\hline 400002 & ACCR & 10000295 & ACT & SUBCN & & \\
\hline 400002 & D002 & 10000032 & ACT & DIRCT & SLC & \\
\hline 400002 & D002 & 10000064 & ACT & SUBCN & & \\
\hline 400002 & D002 & 10000298 & ACT & LABOR & CO00 & \\
\hline 400002 & DO02 & 10000299 & ACT & LABOR & COS & \\
\hline 400002 & D002 & 10000300 & ACT & LABOR & PTB & \\
\hline 400002 & D002 & 10000301 & ACT & LABOR & ABS & \\
\hline 400002 & LIQD & PH000017 & ACT & OVER & MPR & \\
\hline
\end{tabular}

\begin{tabular}{|l|l|l|l|}
\hline ACT & LABOR & COS \\
\hline ACT & LABOR & PTB \\
\hline ACT & LABOR & ABS \\
\hline ACT & OVER & MPR \\
\hline
\end{tabular}

963.00 234.00 36.00 272.16 $6,959.16$ Amount

\begin{tabular}{|c|l|l|l|l|l|l|l|r|}
\hline Proj & Activity & ResourcelD & An Type & Res Type & Category & Amount \\
& & & & & & & \\
\hline 400003 & ACCR & 10000296 & ACT & SUBCN & & $4,000.00$ \\
\hline 400003 & E003 & 10000034 & ACT & DIRCT & SLC & $2,400.00$ \\
\hline 400003 & E003 & 10000091 & ACT & SUBCN & & $3,200.00$ \\
\hline 400003 & E003 & 10000305 & ACT & LABOR & E000 & $4,050.00$ \\
\hline 400003 & E003 & 10000306 & ACT & LABOR & COS & $1,605.00$ \\
\hline 400003 & E003 & 10000307 & ACT & LABOR & PTB & 390.00 \\
\hline 400003 & E003 & 10000308 & ACT & LABOR & ABS & 60.00 \\
\hline 400003 & LIQD & PH000018 & ACT & OVER & MPR & 648.00 \\
\hline
\end{tabular}

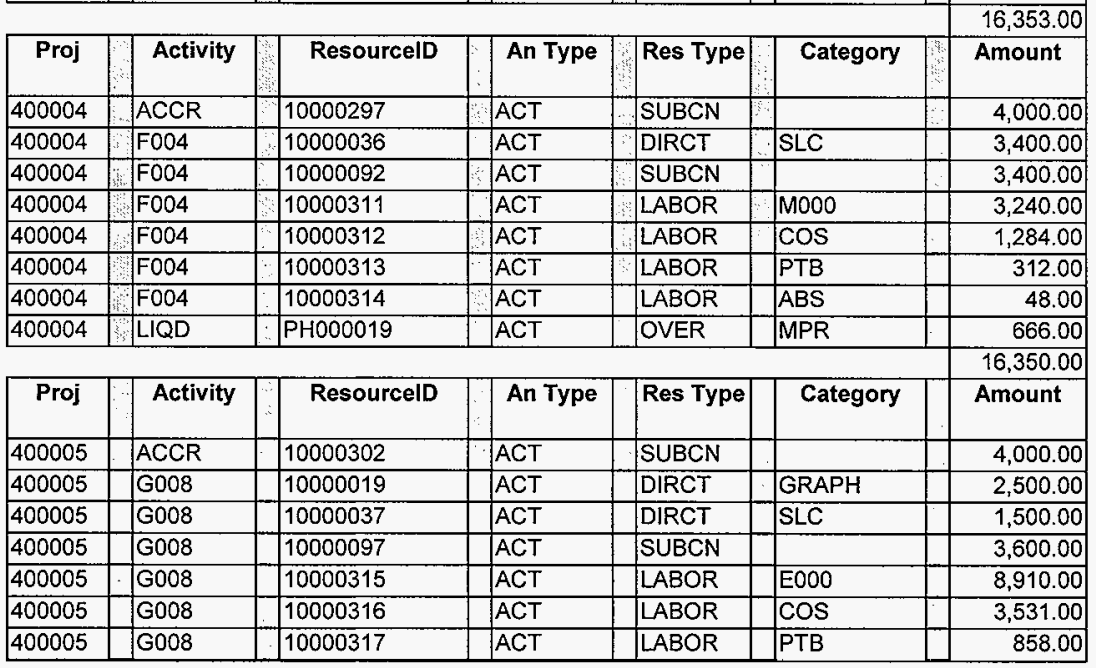




\begin{tabular}{|l|l|l|l|l}
\hline 400005 & G008 & 10000318 & \\
\hline 400005 & LIQD & PH000020 & \\
\hline
\end{tabular}

\begin{tabular}{|l|l|l|l|l|l|}
\hline Proj & Activity & ResourcelD & \\
& & & & \\
\hline 400006 & ACCR & 10000303 & & AC \\
\hline 400006 & K001 & 10000021 & AC \\
\hline 400006 & K001 & 10000038 & AC \\
\hline 400006 & K001 & 10000119 & AC \\
\hline 400006 & K001 & 10000321 & AC \\
\hline 400006 & K001 & 10000322 & AC \\
\hline 400006 & K001 & 10000323 & AC \\
\hline 400006 & K001 & 10000324 & AC \\
\hline 400006 & LIQD & PH000021 & AC \\
\hline
\end{tabular}

\begin{tabular}{|l|l|l|l}
\hline ACT & LABOR & ABS \\
\hline ACT & OVER & AP \\
\hline
\end{tabular}

ABS

132.00 \begin{tabular}{l|l|l}
$A C T$ & OVER & MPR \\
\hline
\end{tabular} 684.00 $25,715.00$

\begin{tabular}{|c|c|c|c|c|c|c|}
\hline & & & & & & $22,159.50$ \\
\hline Proj & Activity & ResourcelD & An Type & Res Type & Category & Amount \\
\hline 500001 & ACCR & 10000304 & ACT & SUBCN & & $4,000.00$ \\
\hline 500001 & LO02 & 10000023 & ACT & DIRCT & GRAPH & $3,500.00$ \\
\hline 500001 & LOO2 & 10000039 & ACT & DIRCT & SLC & $9,000.00$ \\
\hline 500001 & LO02 & 10000073 & ACT & LABOR & M000 & $3,240.00$ \\
\hline 500001 & LO02 & 10000079 & ACT & LABOR & $\cos$ & $1,284.00$ \\
\hline 500001 & LO02 & 10000080 & ACT & LABOR & PTB & 312.00 \\
\hline 500001 & LO02 & 10000081 & ACT & LABOR & ABS & 48.00 \\
\hline 500001 & LO02 & 10000170 & ACT & SUBCN & & $4,000.00$ \\
\hline 500001 & LIQD & $\mathrm{PH} 000022$ & ACT & OVER & MPR & 720.00 \\
\hline & & & & & & $26,104.00$ \\
\hline Proj & Activity & ResourcelD & An Type & Res Type & Category & Amount \\
\hline 500002 & ACCR & 10000309 & ACT & SUBCN & & $4,000.00$ \\
\hline 500002 & LIQD & $\mathrm{PH} 000023$ & ACT & OVER & MPR & 738.00 \\
\hline 500002 & N001 & 10000025 & ACT & DIRCT & GRAPH & $4,000.00$ \\
\hline 500002 & No01 & 10000059 & ACT & SUBCN & & $4,200.00$ \\
\hline 500002 & N001 & 10000082 & ACT & LABOR & $\mathrm{CO00}$ & $3,240.00$ \\
\hline 500002 & No01 & 10000083 & ACT & LABOR & $\cos$ & $1,284.00$ \\
\hline 500002 & N001 & 10000089 & ACT & LABOR & PTB & 312.00 \\
\hline 500002 & N001 & 10000090 & ACT & LABOR & ABS & 48.00 \\
\hline & & & & & & $17,822.00$ \\
\hline Proj & Activity & ResourcelD & An Type & Res Type & Category & Amount \\
\hline 500003 & ACCR & 10000310 & ACT & SUBCN & & $4,000.00$ \\
\hline 500003 & LIQD & $\mathrm{PH} 000024$ & $\overline{A C T}$ & OVER & MPR & 729.00 \\
\hline 500003 & P001 & 10000040 & ACT & DIRCT & SLC & $1,700.00$ \\
\hline 500003 & P001 & 10000093 & ACT & LABOR & E000 & $4,860.00$ \\
\hline 500003 & P001 & 10000094 & ACT & LABOR & $\cos$ & $1,926.00$ \\
\hline 500003 & $P 001$ & 10000095 & ACT & LABOR & PTB & 468.00 \\
\hline 500003 & P001 & 10000096 & ACT & LABOR & ABS & 72.00 \\
\hline
\end{tabular}


PAGE 26 of 32

\begin{tabular}{|c|c|c|c|c|c|c|}
\hline 500003 & P001 & 10000171 & ACT & SUBCN & & $4,100.00$ \\
\hline & & & & & & $17,855.00$ \\
\hline Proj & Activity & ResourcelD & An Type & Res Type & Category & Amount \\
\hline 500004 & $A 001$ & 10000042 & ACT & DIRCT & SLC & 900.00 \\
\hline 500004 & $A 001$ & 10000060 & ACT & SUBCN & & $3,900.00$ \\
\hline 500004 & $A 001$ & 10000098 & ACT & LABOR & MOOO & $2,430.00$ \\
\hline 500004 & $\mathrm{~A} 001$ & 10000104 & ACT & LABOR & $\mathrm{COS}$ & 963.00 \\
\hline 500004 & $\bar{A} 001$ & 10000105 & ACT & LABOR & PTB & 234.00 \\
\hline 500004 & A001 & 10000107 & ACT & LABOR & $A B S$ & 36.00 \\
\hline 500004 & ACCR & 10000319 & ACT & SUBCN & & $4,000.00$ \\
\hline 500004 & LIQD & PH000025 & ACT & OVER & MPR & 711.00 \\
\hline & & & & & & $13,174.00$ \\
\hline Proj & Activity & Resourceld & An Type & Res Type & Category & Amount \\
\hline 500005 & A004 & 10000043 & ACT & DIRCT & SLC & 600.00 \\
\hline 500005 & $A 004$ & 10000110 & $\mathrm{ACT}$ & LABOR & $E 000$ & $2,430.00$ \\
\hline 500005 & A004 & 10000111 & ACT & LABOR & $\cos$ & 963.00 \\
\hline 500005 & $\mathrm{~A} 004$ & 10000112 & ACT & LABOR & PTB & 234.00 \\
\hline 500005 & A004 & 10000117 & ACT & LABOR & ABS & 36.00 \\
\hline 500005 & $\mathrm{~A} 004$ & 10000172 & ACT & SUBCN & & $3,700.00$ \\
\hline 500005 & ACCR & 10000320 & ACT & SUBCN & & $4,000.00$ \\
\hline 500005 & LIQD & $\mathrm{PH} 000026$ & ACT & OVER & MPR & 693.00 \\
\hline & & & & & & $12,656.00$ \\
\hline Proj & Activity & ResourcelD & An Type & Res Type & Category & Amount \\
\hline 500006 & A005 & 10000055 & ACT & DIRCT & SLC & 500.00 \\
\hline 500006 & A005 & 10000121 & ACT & LABOR & M000 & 810.00 \\
\hline 500006 & A005 & 10000122 & ACT & LABOR & $\cos$ & 321.00 \\
\hline 500006 & $\mathrm{~A} 005$ & 10000123 & ACT & LABOR & PTB & 78.00 \\
\hline 500006 & $\mathrm{A005}$ & 10000124 & ACT & LABOR & ABS & 12.00 \\
\hline 500006 & A005 & 10000163 & ACT & SUBCN & & $3,500.00$ \\
\hline 500006 & A05 & 10000044 & ACT & DIRCT & SLC & 500.00 \\
\hline 500006 & ACCR & 10000325 & ACT & SUBCN & & $4,000.00$ \\
\hline 500006 & LIQD & $\mathrm{PH} 000027$ & $A C T$ & OVER & MPR & 675.00 \\
\hline & & & & & & $10,396.00$ \\
\hline Proj & Activity & ResourcelD & An Type & Res Type & Category & Amount \\
\hline 500007 & ACCR & 10000326 & ACT & SUBCN & & $4,000.00$ \\
\hline 500007 & D002 & 10000045 & $\mathrm{ACT}$ & DIRCT & SLC & $1,100.00$ \\
\hline 500007 & D002 & 10000120 & ACT & SUBCN & & $3,300.00$ \\
\hline 500007 & D002 & 10000131 & ACT & LABOR & E000 & $4,050.00$ \\
\hline 500007 & $\mathrm{D} 002$ & 10000132 & ACT & LABOR & $\cos$ & $1,605.00$ \\
\hline 500007 & D002 & 10000133 & ACT & LABOR & PTB & 390.00 \\
\hline 500007 & D002 & 10000134 & ACT & LABOR & ABS & 60.00 \\
\hline 500007 & LIQD & $\mathrm{PH} 000028$ & ACT & OVER & MPR & 657.00 \\
\hline & & & & & & $15,162.00$ \\
\hline
\end{tabular}


PAGE 27 of 32

\begin{tabular}{|c|l|l|l|l|l|}
\hline Proj & Activity & ResourcelD & A \\
& & & \\
\hline 500008 & ACCR & 10000327 & AC \\
\hline 500008 & E003 & 10000046 & AC \\
\hline 500008 & E003 & 10000129 & AC \\
\hline 500008 & E003 & 10000141 & AC \\
\hline 500008 & E003 & 10000142 & AC \\
\hline 500008 & EO03 & 10000143 & AC \\
\hline 500008 & EO03 & 10000144 & $A C$ \\
\hline 500008 & LIQD & PH000029 & AC \\
\hline
\end{tabular}

\begin{tabular}{|l|l|l|l|}
\hline An Type & Res Type & Category \\
\hline ACT & SUBCN & \\
\hline ACT & DIRCT & SLC \\
\hline ACT & SUBCN & \\
\hline ACT & LABOR & EO00 \\
\hline ACT & LABOR & COS \\
\hline ACT & LABOR & PTB \\
\hline ACT & LABOR & ABS \\
\hline ACT & OVER & MPR \\
\hline
\end{tabular}

Amount

\begin{tabular}{|c|l|l|l|l|}
\hline Proj & Activity & ResourcelD & \\
& & & & \\
\hline 500009 & ACCR & 10000328 & & $A C$ \\
\hline 500009 & F004 & 10000072 & \\
\hline 500009 & F004 & 10000152 & & $A C$ \\
\hline 500009 & F004 & 10000153 & & $A C$ \\
\hline 500009 & F004 & 10000154 & & $A C$ \\
\hline 500009 & F004 & 10000156 & \\
\hline 500009 & LIQD & PH000030 & AC \\
\hline
\end{tabular}

\begin{tabular}{|l|l|l}
\hline An Type & \\
\hline ACT & \\
\hline ACT & \\
\hline ACT & \\
\hline ACT & \\
\hline ACT & \\
\hline ACT & \\
\hline ACT & \\
\hline
\end{tabular}

\begin{tabular}{|l|l|}
\hline Res Type \\
\hline SUBCN \\
\hline SUBCN \\
\hline LABOR \\
\hline LABOR \\
\hline LABOR \\
\hline LABOR \\
\hline OVER \\
\hline
\end{tabular}

\section{Category}

Pro

\begin{tabular}{l|l|l}
\hline 500010 & ACCR & 10000329 \\
\hline
\end{tabular}

$500010 \quad$ G008

\begin{tabular}{l|l}
\hline 500010 & G008
\end{tabular}

\begin{tabular}{|l:l}
\hline 500010 & G008 \\
\hline 500010 & G008
\end{tabular}

\begin{tabular}{|l|l}
\hline 500010 & G008 \\
\hline
\end{tabular}

\begin{tabular}{l:l}
\hline 500010 & G008 \\
\hline 500010 & G008
\end{tabular}

\begin{tabular}{|l|l}
\hline 500010 & G008 \\
\hline
\end{tabular}

$500010 \quad$ LIQD

\begin{tabular}{|l|l|l|}
\hline & ResourcelD \\
\hline 10000329 & \\
\hline 10000047 & \\
\hline 10000166 & & \\
\hline 10000167 & \\
\hline 10000168 & \\
\hline 10000169 & \\
\hline 10000346 & \\
\hline PH000031 & AC \\
\hline
\end{tabular}

An Type

ACT

$\mathrm{ACT}$

ACT

ACT

ACT

ACT

ACT

ACT

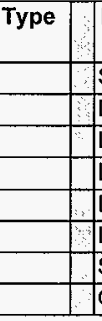

\section{Res Type}

Category

Amount

$14,623.00$

\begin{tabular}{|c|c|c|c|c|c|c|}
\hline & & & & & & $22,902.50$ \\
\hline Proj & Activity & ResourcelD & An Type & Res Type & Category & Amount \\
\hline 500011 & ACCR & 10000330 & ACT & SUBCN & & $4,000.00$ \\
\hline 500011 & K001 & 10000048 & ACT & DIRCT & SLC & 200.00 \\
\hline 500011 & K001 & 10000099 & $\overline{A C T}$ & SUBCN & & $7,000.00$ \\
\hline 500011 & K001 & 10000174 & ACT & LABOR & E000 & $3,240.00$ \\
\hline 500011 & K001 & 10000175 & $\overline{A C T}$ & LABOR & $\cos$ & $1,284.00$ \\
\hline 500011 & K001 & 10000180 & ACT & LABOR & PTB & 312.00 \\
\hline 500011 & K001 & 10000181 & ACT & LABOR & ABS & 48.00 \\
\hline 500011 & LIQD & $\mathrm{PH} 000032$ & ACT & OVER & MPR & 990.00 \\
\hline & & & & & & $17,074.00$ \\
\hline Proj & Activity & ResourcelD & An Type & Res Type & Category & Amount \\
\hline 500012 & ACCR & 10000331 & ACT & SUBCN & & $4,000.00$ \\
\hline 500012 & $L .002$ & 10000106 & $A C \bar{T}$ & SUBCN & & $7,500.00$ \\
\hline
\end{tabular}


PAGE 28 of 32

\begin{tabular}{|c|c|c|c|c|c|c|}
\hline 500012 & L002 & 10000182 & ACT & LABOR & M000 & $3,645.00$ \\
\hline 500012 & L002 & 10000183 & ACT & LABOR & $\cos$ & $1,444.50$ \\
\hline 500012 & L002 & 10000184 & $\overline{A C T}$ & LABOR & PTB & 351.00 \\
\hline 500012 & LOO2 & 10000185 & ACT & LABOR & ABS & 54.00 \\
\hline 500012 & LIQD & $\mathrm{PH} 000033$ & ACT & OVER & MPR & $1,035.00$ \\
\hline & & & & & & $18,029.50$ \\
\hline Proj & Activity & ResourcelD & An Type & Res Type & Category & Amount \\
\hline 500013 & ACCR & 10000332 & ACT & SUBCN & & $4,000.00$ \\
\hline 500013 & LIQD & $\mathrm{PH} 000034$ & ACT & OVER & MPR & $1,080.00$ \\
\hline 500013 & No01 & 10000061 & ACT & SUBCN & & $8,000.00$ \\
\hline 500013 & N001 & 10000187 & ACT & LABOR & $\mathrm{CO00}$ & $3,645.00$ \\
\hline 500013 & N001 & 10000188 & ACT & LABOR & $\cos$ & $1,444.50$ \\
\hline 500013 & No01 & 10000189 & ACT & LABOR & PTB & 351.00 \\
\hline 500013 & N001 & 10000190 & ACT & LABOR & ABS & 54.00 \\
\hline & & & & & & $18,574.50$ \\
\hline Proj & Activity & ResourcelD & An Type & Res Type & Category & Amount \\
\hline 500014 & ACCR & 10000333 & ACT & SUBCN & & $4,000.00$ \\
\hline 500014 & LIQD & PH000035 & ACT & OVER & MPR & $1,125.00$ \\
\hline 500014 & P001 & 10000146 & ACT & SUBCN & & $8,500.00$ \\
\hline 500014 & P001 & 10000194 & ACT & LABOR & E000 & $8,100.00$ \\
\hline 500014 & P001 & 10000195 & ACT & LABOR & $\cos$ & $3,210.00$ \\
\hline 500014 & $P 001$ & 10000196 & ACT & LABOR & PTB & 780.00 \\
\hline 500014 & P001 & 10000197 & ACT & LABOR & ABS & 120.00 \\
\hline & & & & & & $25,835.00$ \\
\hline Proj & Activity & ResourcelD & An Type & Res Type & Category & Amount \\
\hline 500015 & $A 001$ & 10000049 & ACT & DIRCT & SLC & $1,500.00$ \\
\hline 500015 & $A 001$ & 10000151 & ACT & SUBCN & & $9,000.00$ \\
\hline 500015 & A001 & 10000202 & ACT & LABOR & $E 000$ & $8,100.00$ \\
\hline 500015 & A001 & 10000203 & ACT & LABOR & $\cos$ & $3,210.00$ \\
\hline 500015 & $\mathrm{~A} 001$ & 10000204 & ACT & LABOR & PTB & 780.00 \\
\hline 500015 & A001 & 10000205 & ACT & LABOR & $\overline{A B S}$ & 120.00 \\
\hline 500015 & ACCR & 10000334 & ACT & SUBCN & & $4,000.00$ \\
\hline 500015 & LIQD & $\mathrm{PH} 000036$ & ACT & OVER & MPR & $1,170.00$ \\
\hline & & & & & & $27,880.00$ \\
\hline Proj & Activity & ResourcelD & An Type & Res Type & Category & Amount \\
\hline 800001 & A004 & 10000002 & ACT & DIRCT & OCCUP & $2,500.00$ \\
\hline 800001 & $A 004$ & 10000028 & ACT & DIRCT & GRAPH & $4,500.00$ \\
\hline 800001 & A004 & 10000050 & $A C T$ & DIRCT & SLC & 300.00 \\
\hline 800001 & A004 & 10000078 & ACT & SUBCN & & $9,500.00$ \\
\hline 800001 & A004 & 10000217 & ACT & LABOR & $E 000$ & $3,240.00$ \\
\hline 800001 & A004 & 10000218 & ACT & LABOR & $\cos$ & $1,284.00$ \\
\hline 800001 & A004 & 10000219 & ACT & LABOR & PTB & 312.00 \\
\hline 800001 & A004 & 10000220 & ACT & LABOR & ABS & 48.00 \\
\hline
\end{tabular}


HANDI Fluor Daniel Hanford - PeopleSoft Financials

FIT GAP ANALYSIS

HNF-2746 REV 0

April 17, 1998

PAGE 29 of 32

800001

ACCR

10000335

800001

LIQD

PH000037

ACT

ACT

SUBCN

OVER

MPR

$4,000.00$




\begin{tabular}{|c|l|l|l|l|l|l|l|l|}
\hline Proj & Activity & ResourcelD & An Type & Res Type & Category \\
\hline 800002 & A004 & 10000003 & ACT & DIRCT & OCCUP \\
\hline 800002 & A005 & 10000051 & ACT & DIRCT & SLC & \\
\hline 800002 & A005 & 10000173 & ACT & SUBCN & & \\
\hline 800002 & A005 & 10000222 & ACT & LABOR & MO00 \\
\hline 800002 & A005 & 10000223 & ACT & LABOR & COS \\
\hline 800002 & A005 & 10000224 & ACT & LABOR & PTB \\
\hline 800002 & A005 & 10000225 & ACT & LABOR & ABS \\
\hline 800002 & ACCR & 10000336 & ACT & SUBCN & & \\
\hline 800002 & LIQD & PH000038 & ACT & OVER & MPR \\
\hline
\end{tabular}

Amount

\begin{tabular}{|c|l|l|l|}
\hline Proj & Activity & \\
\hline 800003 & & A004 & 100 \\
\hline 800003 & ACCR & \\
\hline 800003 & D002 & \\
\hline 800003 & D002 & \\
\hline 800003 & D002 & \\
\hline 800003 & D002 & \\
\hline 800003 & D002 & \\
\hline 800003 & LIQD & PH \\
\hline
\end{tabular}

\begin{tabular}{|l|l|l|l|}
\hline ResourcelD & An Type & R & \\
\hline 10000004 & ACT & D \\
\hline 10000337 & ACT & S \\
\hline 10000062 & ACT & S \\
\hline 10000234 & ACT & LA \\
\hline 10000235 & ACT & L \\
\hline 10000236 & ACT & L \\
\hline 10000237 & ACT & L \\
\hline PH000039 & ACT & OCT \\
\hline
\end{tabular}

\begin{tabular}{|l|l|l|}
\hline Res Type & Category \\
\hline DIRCT & OCCUP \\
\hline SUBCN & \\
\hline SUBCN & \\
\hline LABOR & C000 \\
\hline LABOR & COS \\
\hline LABOR & PTB \\
\hline LABOR & ABS \\
\hline OVER & MPR \\
\hline
\end{tabular}

$2,500.00$

$1,100.00$

$10,000.00$

$3,240.00$

$1,284.00$ 312.00 48.00 $4,000.00$

$1,260.00$ $23,744.00$

\begin{tabular}{|c|c|c|c|c|c|c|}
\hline Proj & Activity & ResourcelD & An Type & Res Type & Category & Amount \\
\hline 900001 & A004 & 10000007 & ACT & DIRCT & OCCUP & $3,000.00$ \\
\hline 900001 & ACCR & 10000338 & ACT & SUBCN & & $4,000.00$ \\
\hline 900001 & E003 & 10000033 & ACT & DIRCT & GRAPH & $5,000.00$ \\
\hline 900001 & E003 & 10000063 & ACT & SUBCN & & $12,000.00$ \\
\hline 900001 & E003 & 10000230 & ACT & LABOR & E000 & $5,670.00$ \\
\hline 900001 & E003 & 10000231 & ACT & LABOR & $\overline{\cos }$ & $2,247.00$ \\
\hline 900001 & E003 & 10000232 & ACT & LABOR & PTB & 546.00 \\
\hline 900001 & E003 & 10000233 & ACT & LABOR & ABS & 84.00 \\
\hline 900001 & LIQD & PH000040 & ACT & OVER & $\overline{M P R}$ & $1,440.00$ \\
\hline & & & & & & $33,987.00$ \\
\hline Proj & Activity & ResourcelD & An Type & Res Type & Category & Amount \\
\hline 900002 & $\mathrm{A004}$ & 10000008 & $A C T$ & DIRCT & OCCUP & $3,500.00$ \\
\hline 900002 & ACCR & 10000339 & ACT & SUBCN & & $4,000.00$ \\
\hline 900002 & F004 & 10000052 & ACT & DIRCT & SLC & 300.00 \\
\hline 900002 & $F 004$ & 10000065 & ACT & SUBCN & & $13,000.00$ \\
\hline 900002 & F004 & 10000238 & ACT & LABOR & M000 & $6,480.00$ \\
\hline 900002 & F004 & 10000239 & $A C T$ & LABOR & $\cos$ & $2,568.00$ \\
\hline 900002 & F004 & 10000240 & ACT & LABOR & PTB & 624.00 \\
\hline 900002 & F004 & 10000241 & ACT & LABOR & ABS & 96.00 \\
\hline 900002 & LIQD & $\mathrm{PHO00041}$ & ACT & OVER & MPR & $1,530.00$ \\
\hline
\end{tabular}


PAGE 31 of 32

$32,098.00$

\begin{tabular}{|c|c|c|c|c|c|c|}
\hline Proj & Activity & ResourcelD & An Type & Res Type & Category & Amount \\
\hline 900003 & G008 & 10000242 & ACT & LABOR & $E 000$ & $6,885.00$ \\
\hline 900003 & ACCR & 10000340 & ACT & SUBCN & & $4,000.00$ \\
\hline 900003 & $\mathrm{~A} 004$ & 10000009 & ACT & DIRCT & OCCUP & $3,500.00$ \\
\hline 900003 & G008 & 10000243 & ACT & LABOR & $\cos$ & $2,728.50$ \\
\hline 900003 & G008 & 10000176 & ACT & SUBCN & & 900.00 \\
\hline 900003 & G008 & 10000244 & ACT & LABOR & PTB & 663.00 \\
\hline 900003 & LIQD & $\mathrm{PH} 000048$ & ACT & OVER & MPR & 441.00 \\
\hline 900003 & G008 & 10000245 & ACT & LABOR & ABS & 102.00 \\
\hline 900003 & LIQD & $\mathrm{PH} 000043$ & $\mathrm{ACT}$ & DIRCT & ABS & -156.27 \\
\hline 900003 & LIQD & $\mathrm{PH} 000047$ & $A C T$ & DIRCT & PTB & $-1,015.74$ \\
\hline 900003 & LIQD & $\mathrm{PH} 000044$ & ACT & DIRCT & $\cos$ & $-4,180.29$ \\
\hline 900003 & LIQD & $\mathrm{PH} 000046$ & ACT & DIRCT & OCCUP & $-5,362.23$ \\
\hline 900003 & LIQD & $\mathrm{PH} 000042$ & ACT & DIRCT & & $-7,507.08$ \\
\hline 900003 & LIQD & $\mathrm{PH} 000045$ & $A C T$ & DIRCT & E000 & $-10,548.15$ \\
\hline & & & & & & $-9,550.26$ \\
\hline Proj & Activity & ResourcelD & An Type & Res Type & Category & Amount \\
\hline 900004 & A004 & 10000010 & ACT & DIRCT & OCCUP & $4,000.00$ \\
\hline 900004 & $\mathrm{~A} 004$ & 10000011 & ACT & DIRCT & OCCUP & $4,000.00$ \\
\hline 900004 & ACCR & 10000341 & ACT & SUBCN & & $4,000.00$ \\
\hline 900004 & ACCR & 10000342 & ACT & SUBCN & & $4,000.00$ \\
\hline 900004 & K001 & 10000067 & ACT & SUBCN & & $1,500.00$ \\
\hline 900004 & K001 & 10000177 & ACT & SUBCWN & & $1,200.00$ \\
\hline 900004 & K001 & 10000250 & ACT & LABOR & E000 & $6,480.00$ \\
\hline 900004 & K001 & 10000251 & ACT & LABOR & $\cos$ & $2,568.00$ \\
\hline 900004 & K001 & 10000252 & ACT & LABOR & PTB & 624.00 \\
\hline 900004 & K001 & 10000253 & $\mathrm{ACT}$ & LABOR & ABS & 96.00 \\
\hline 900004 & K001 & 10000254 & ACT & LABOR & $\mathrm{C000}$ & $6,480.00$ \\
\hline 900004 & K001 & 10000255 & ACT & LABOR & $\cos$ & $2,568.00$ \\
\hline 900004 & K001 & 10000256 & ACT & LABOR & PTB & 624.00 \\
\hline 900004 & K001 & 10000257 & ACT & LABOR & ABS & 96.00 \\
\hline 900004 & LIQD & PH000049 & ACT & OVER & MPR & 495.00 \\
\hline 900004 & LIQD & PH000050 & ACT & OVER & MPR & 468.00 \\
\hline & & & & & & $39,199.00$ \\
\hline Proj & Activity & ResourcelD & An Type & Res Type & Category & Amount \\
\hline 900005 & A004 & 10000014 & ACT & DIRCT & OCCUP & $31,000.00$ \\
\hline 900005 & ACCR & 10000343 & ACT & SUBCN & & $4,000.00$ \\
\hline 900005 & L002 & 10000178 & ACT & SUBCN & & $1,800.00$ \\
\hline 900005 & L002 & 10000263 & $A C T$ & LABOR & MO00 & $8,100.00$ \\
\hline 900005 & L002 & 10000264 & $A C T$ & LABOR & $\cos$ & $3,210.00$ \\
\hline 900005 & LO02 & 10000265 & $A C T$ & LABOR & PTB & 780.00 \\
\hline 900005 & L002 & 10000266 & $A C T$ & LABOR & ABS & 120.00 \\
\hline 900005 & LIQD & PHO00051 & ACT & OVER & MPR & 522.00 \\
\hline
\end{tabular}




\begin{tabular}{|c|c|c|c|c|c|c|}
\hline & & & & & & $49,532.00$ \\
\hline Proj & Activity & ResourcelD & An Type & Res Type & Category & Amount \\
\hline 900006 & A004 & 10000012 & ACT & DIRCT & OCCUP & $5,000.00$ \\
\hline 900006 & ACCR & 10000344 & ACT & SUBCN & 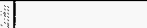 & $4,000.00$ \\
\hline 900006 & LIQD & $\mathrm{PH} 000052$ & ACT & OVER & MPR & 549.00 \\
\hline 900006 & N001 & 10000068 & ACT & SUBCN & & $2,100.00$ \\
\hline 900006 & N001 & 10000267 & ACT & LABOR & C000 & $3,240.00$ \\
\hline 900006 & N001 & 10000268 & ACT & LABOR & $\cos$ & $1,284.00$ \\
\hline 900006 & N001 & 10000269 & ACT & LABOR & PTB & 312.00 \\
\hline 900006 & N001 & 10000270 & ACT & LABOR & ABS & 48.00 \\
\hline 900006 & P001 & 10000053 & $A C T$ & DIRCT & SLC & $47,800.00$ \\
\hline & & & & & & $64,333.00$ \\
\hline Proj & Activity & ResourcelD & An Type & Res Type & Category & Amount \\
\hline 900007 & ACCR & 10000345 & ACT & SUBCN & & $4,000: 00$ \\
\hline 900007 & LIQD & $\mathrm{PH} 000053$ & ACT & OVER & MPR & 765.00 \\
\hline 900007 & P001 & 10000035. & ACT & DIRCT & GRAPH & $27,000.00$ \\
\hline 900007 & P001 & 10000069 & ACT & SUBCN & & $4,500.00$ \\
\hline 900007 & P001 & 10000275 & ACT & LABOR & MOO0 & $3,240.00$ \\
\hline 900007 & P001 & 10000276 & ACT & LABOR & $\cos$ & $1,284.00$ \\
\hline 900007 & P001 & 10000277 & ACT & LABOR & PTB & 312.00 \\
\hline 900007 & P001 & 10000278 & ACT & LABOR & ABS & 48.00 \\
\hline & & & & & & $41,149.00$ \\
\hline Proj & Activity & ResourcelD & An Type & Res Type & Category & Amount \\
\hline 900008 & $\mathrm{AO4}$ & 10000179 & ACT & MATER & & $1,000.00$ \\
\hline 900008 & LIQD & PH000054 & ACT & OVER & MPR & 90.00 \\
\hline
\end{tabular}

